\title{
Robustness and fragility of Boolean models for genetic regulatory networks
}

\author{
${\text { Madalena } \text { Chaves }^{1 *} \text {, Réka Albert }}^{2}$ \& Eduardo D. Sontag ${ }^{1}$ \\ ${ }^{1}$ Department of Mathematics and BioMaPS Institute for Quantitative Biology, \\ Rutgers University, Piscataway, NJ 08854 \\ ${ }^{2}$ Department of Physics and Huck Institutes for the Life Sciences, \\ Pennsylvania State University, University Park, PA 16802. \\ *Corresponding author, madalena@math.rutgers.edu
}

\begin{abstract}
Interactions between genes and gene products give rise to complex circuits that enable cells to process information and respond to external signals. Theoretical studies often describe these interactions using continuous, stochastic, or logical approaches. We propose a new modeling framework for gene regulatory networks, that combines the intuitive appeal of a qualitative description of gene states with a high flexibility in incorporating stochasticity in the duration of cellular processes. We apply our methods to the regulatory network of the segment polarity genes, thus gaining novel insights into the development of gene expression patterns. For example, we show that very short synthesis and decay times can perturb the wild type pattern. On the other hand, separation of timescales between pre- and posttranslational processes and a minimal prepattern ensure convergence to the wild type expression pattern regardless of fluctuations.
\end{abstract}

Keywords: Gene regulatory networks; segment polarity genes; Boolean models.

\section{Introduction}

Understanding how genetic information is translated into proteins to produce various cell types remains a major challenge in contemporary biology [Wolpert et al., 1998]. Gene products often regulate the synthesis of mRNAs and proteins, forming complex networks of regulatory interactions. Concurrently with experimental progress in gene control networks [Davidson et al., 2002], several alternative modeling frameworks have been proposed. In the continuous-state approach, the concentrations of cellular components are assumed to be continuous functions of time, governed by differential equations with mass-action (or more general) kinetics [Reinitz and Sharp, 1995, von Dassow et al., 2000, Gursky et al., 2001]. Stochastic models address the deviations from population homogeneity by transforming reaction rates into probabilities and concentrations into numbers of molecules [Rao et al., 2002]. Finally, in the discrete approach, each component is assumed to have a small number of qualitative states, and the regulatory interactions are described by logical functions [Mendoza et al., 1999, Sánchez and Thieffry, 2001 Yuh et al., 2001, Kauffman et al., 2003 Ghysen and Thomas, 2003 Bodnar, 1997 Albert and Othmer, 2003].

The kinetic details of protein-protein or protein-DNA interactions are rarely known, but there is increasing evidence that the input-output curves of regulatory relationships are strongly sigmoidal and can be 
well approximated by step functions [Yuh et al., 2001 Thomas, 1973]. Moreover, both models and experiments suggest that regulatory networks are remarkably robust, that is, they maintain their function even when faced with fluctuations in components and reaction rates von Dassow et al., 2000 Alon et al., 1999. Eldar et al., 2002, Carlson and Doyle, 2002, Conant and Wagner, 2004]. These observations lend support to the assumption of discrete states for genetic network components and of combinatorial rules for the effects of transcription factors [Glass and Kauffman, 1973, de Jong et al., 2004]. The extreme of discretization, Boolean models, consider only two states (expressed or not), closely mimicking the inference methods used in genetics [Kauffman et al., 2003, Thomas, 1973, Kauffman, 1993]. It is straightforward to study the effect of knock-out mutations or changes in initial conditions in this framework, and the agreement between a real system and a Boolean model of it is a strong indication of the robustness of the system to changes in kinetic details [Albert and Othmer, 2003].

In discrete models the decision whether a network node (component) will be affected by a synthesis or decay process is determined by the state of effector nodes (nodes that interact with it). Typical timedependent Boolean models use synchronous updating rules [Kauffman et al., 2003 Albert and Othmer, 2003 Bodnar, 1997 Kauffman, 1993, assuming that the time scales of the processes taking place in the system are similar. In reality the timescales of transcription, translation, and degradation can vary widely from gene to gene and can be anywhere from minutes to hours. Logical models following the formalism introduced by René Thomas [Thomas, 1973] allow asynchronism by associating two variables to each gene: a state variable describing the level of its protein, and an image variable that is the output of the logical rule whose inputs are the state variables of effector nodes. Whether the future state variable of a gene equals the image or current state variable depends on the update order and, in the absence of temporal information, the Thomas formalism focuses on determining the steady states, where the state and image variables coincide [Mendoza et al., 1999, Sánchez and Thieffry, 2001 Ghysen and Thomas, 2003 Bernot et al., 2004]. The effect of asynchronous updates on the dynamics of the system, however, has not been explored yet.

In this paper, we present a methodology for testing the robustness of Boolean models with respect to stochasticity in the order of updates. Through this, we are also probing the system itself: will individual variations lead to unexpected gene expression patterns? In the asynchronous method, the synthesis/decay decision is made at different time-points for each node, allowing individual variability in each process' duration, but more importantly, it allows for decision reversal if the dynamics of effector nodes changes. It becomes possible to reproduce, e.g., the overturning of mRNA decay when its transcriptional activator is synthesized, a process that synchronous update cannot capture. Thus, replacing synchronous with asynchronous updates is not merely a technical detail, but rather a fundamental paradigm shift from pointwise in time to potentially continuous communication between nodes. Indeed, the effective synthesis or decay time for a certain node are determined by the time interval between the latest update of its effector nodes and its current update time, and can be any positive fraction of the unit time interval. We propose three algorithms, with varying freedom in the relative duration of cellular processes, and find that very short transcription or decay times have the potential to derail the wild type development process.

The steady states of a Boolean model will remain the same regardless of the mechanism of update, but its dynamical behavior can be drastically altered due to the stochastic nature of the updates; for instance, the same initial state may lead to different steady states or limit cycles. Since the duration of synthesis and decay processes is not known, we randomly explore the space of all possible timescales and update orders, and derive the probability of different outcomes. Our methods offer a systematic way of exploring generic behavior of gene regulatory networks and comparing it to experimentally observed outcomes. To present a concrete example, we generalize a previously introduced Boolean model of the Drosophila segment polarity genes [Albert and Othmer, 2003]. This model reproduces the wild type steady state pattern of the segment 


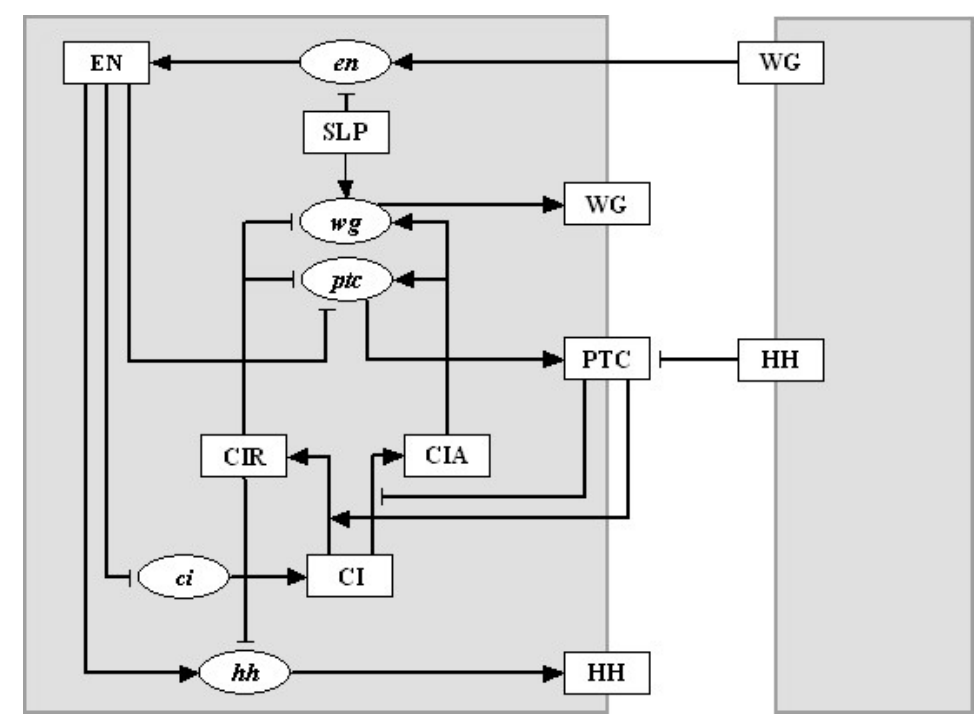

Figure 1: The network of interactions between the segment polarity genes. The grey background layers illustrate two neighboring cells, indicating that some interactions in this network are inter-cellular. The shape of the nodes indicates whether the corresponding substances are mRNAs (ellipses) or proteins (rectangles). The edges of the network signify either biochemical reactions (e.g. translation,protein interactions) or regulatory interactions (e.g. transcriptional activation). The edges are classified as activating $(\rightarrow$ ) or inhibiting $(\dashv)$. Figure adapted from [Albert and Othmer, 2003].

polarity genes as well as the gene patterns of mutants, but its dynamic behavior is not directly comparable to that of the real system. Here we show that asynchronous update leads to a much more realistic model that gives further insights into the robustness of the gene regulatory network.

\section{The segment polarity gene network in Drosophila}

The Drosophila melanogaster segment polarity genes represent the last step in the hierarchical cascade of gene families initiating the segmented body of the fruit fly. While the preceding genes act transiently, the segment polarity genes are expressed throughout the life of the fly, and their periodic spatial pattern is maintained for at least 3 hours of embryonic development [Wolpert et al., 1998]. The regulatory roles of the previously expressed genes such as the pair-rule genes fushi tarazu, runt, even-skipped are incorporated in the prepattern (initial state) of the segment polarity genes. The stable maintenance of the segment polarity gene expression is due to the interactions between these genes (see Figure 1), and it is a crucial requirement in the development and stability of the parasegmental furrows. The best characterized segment polarity genes include engrailed (en), wingless (wg), hedgehog ( $h h$ ), patched (ptc), cubitus interruptus (ci) and sloppy paired $(s l p)$, encoding for diverse proteins including transcription factors as well as secreted and receptor proteins.

The pair-rule gene sloppy paired $(s l p)$ is activated before the segment polarity genes and expressed constitutively thereafter [Grossniklaus et al., 1992, Cadigan et al., 1994]. slp encodes two forkhead domain transcription factors with similar functions that activate $w g$ transcription and repress en transcription, and since they are co-expressed we designate them both SLP. The $w g$ gene encodes a glycoprotein that is secreted from the cells that synthesize it [Hooper and Scott, 1992 [Pfeiffer \& Vincent 1999], and can bind to the Frizzled receptor on neighboring cells, initiating a signaling cascade leading to the transcription of engrailed (en) [Cadigan \& Nusse 1997]. EN, the homeodomain-containing product of the en gene, pro- 
motes the transcription of the hedgehog gene ( $h h$ ) [Tabata et al., 1992]. In addition to the homeodomain, EN contains a separate repression domain that affects the transcription of $c i$ [Eaton \& Kornberg 1990] and possibly ptc [Hidalgo and Ingham, 1990, Taylor et al., 1993]. The hedgehog protein (HH) is tethered to the cell membrane by a cholesterol linkage that is severed by the dispatched protein, freeing it to bind to the $\mathrm{HH}$ receptor PTC on a neighboring cell [Ingham \& McMahon 2001]. The intracellular domain of PTC forms a complex with smoothened (SMO) in which SMO is inactivated by a post-translational conformation change (Ingham 1998). Binding of HH to PTC removes the inhibition of SMO, and activates a pathway that results in the modification of CI [Ingham 1998]. The CI protein can be converted into one of two transcription factors, depending on the PTC-HH interactions. In the absence of $\mathrm{HH}$ signaling $\mathrm{CI}$ is cleaved to form CIR, a transcriptional repressor that represses $w g, p t c$ and $h h$ transcription[Aza-Blanc \& Kornberg 1999]. When secreted HH binds to PTC and frees SMO, CI is converted to a transcriptional activator, CIA, that promotes the transcription of $w g$ and ptc [Aza-Blanc \& Kornberg 1999 Ohlmeyer \& Kalderon 1998].

The initial state of the Drosophila segment polarity genes includes two-cell-wide SLP stripes followed by two-cell-wide stripes not expressing SLP [Cadigan et al., 1994], single-cell-wide $w g$, en and hh stripes followed by three cells not expressing them, and three-cell-wide stripes for $c i$ and $p t c$ [Hooper and Scott, 1992 Wolpert et al., 1998]: This pattern is maintained almost unmodified for three hours ${ }^{1}$ (see Fig. 2a), during which time the embryo is divided into 14 parasegments by furrows positioned between the the $w g$ and $e n$ -expressing cells [Hooper and Scott, 1992].

The first model of the segment polarity gene network was proposed by von Dassow and collaborators von Dassow et al., 2000, and is a continuous-state model of 13 equations and 48 unknown kinetic parameters. The main conclusion of the [von Dassow et al., 2000] article is that the gene patterns are robust with respect to variations in the kinetic constants in the rate laws, thus the essential feature of this network is its topology, i.e. the existence and signature (activating or inhibiting) of the interactions. The idea of the network topology determining its dynamics was further explored by [Albert and Othmer, 2003], who used a slightly different network reconstruction and assumed synchronous Boolean regulation among nodes. In the Albert and Othmer, 2003 model each mRNA and protein is represented by a node of a network, and the state of each node is 1 or 0 , according to whether the corresponding substance is present or not. The states of the nodes are updated synchronously, and the future state of node $i$ is determined by a Boolean function of its current state and the current states of those nodes that have edges incident on it. The updating functions are based on the experimental information and on the following dynamical assumptions: (i) the synthesis of mRNAs/proteins has the duration of one timestep; (ii) the effect of transcriptional activators and inhibitors is never additive, but rather, inhibitors are dominant; (iii) mRNAs decay in one timestep if not transcribed; (iv) transcription factors and proteins undergoing post-translational modification decay in one timestep if their mRNA is not present; (v) protein-protein binding, such as in the formation of the Patched-Hedgehog complex, is assumed to be instantaneous. In summary, the [Albert and Othmer, 2003] model assumes that gene transcription, protein translation, mRNA and protein decay all happen on a similar timescale, while protein complex formation is instantaneous compared to this common timescale.

The von Dassow et al., 2000] and [Albert and Othmer, 2003] models agree in their conclusions regarding the robustness of the segment polarity gene network. The simplicity of the Boolean rules in the latter also allows for the exploration of knock-out mutations and changes in the prepattern of the segment polarity genes. Starting from the known initial state of en, wg, hh, ptc, $c i$ and SLP, and assuming the null(off) state for all other nodes the Albert and Othmer, 2003 model leads to a time-invariant spatial pattern (see Fig. 2a) that coincides with the experimentally observed wild-type expression of the segment polarity genes

\footnotetext{
${ }^{1}$ A notable exception includes the refinement of the $p t c$ pattern.
} 

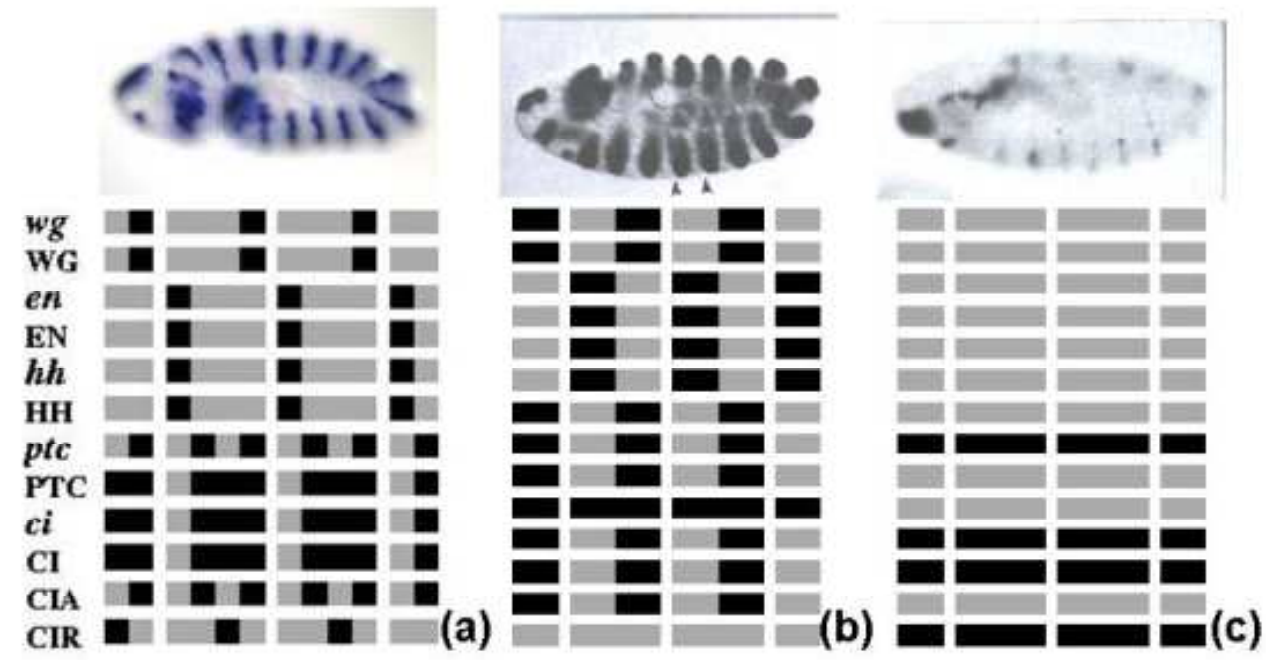

Figure 2: a) Top: Illustration of the gene expression pattern of wingless on a gastrulating (stage 9) embryo. Other segment polarity genes have similar periodic patterns that are maintained for around three hours of embryonic development. The parasegmental furrows form at the posterior border of the $w g$ expressing cells [Wolpert et al., 1998]. Bottom: Synthesis of the wild type expression patterns of the segment polarity genes (see also text) [Hooper and Scott, 1992] Wolpert et al., 1998]. Left corresponds to anterior and right to posterior in each parasegment. Horizontal rows correspond to the pattern of individual nodes - specified at the left side of the row - over two full and two partial parasegments. Each parasegment is assumed to be four cells wide. A black (gray) box denotes a node that is (is not) expressed. b) Top: wingless expression pattern in an patched knock-out mutant embryo at stage 11 [Tabata et al., 1992]. The wingless stripes broaden, and secondary furrows appear at the middle of the parasegment, indicating a new en-wg boundary. Bottom: Broad striped steady state of the Boolean model, obtained when patched is kept off (with the change that $p t c$ and PTC are not expressed), or when $w g$, en, hh are initiated in every cell [Albert and Othmer, 2003]. This steady state agrees with all experimental observations on ptc mutants and heat-shocked genes [Tabata et al., 1992 Gallet et al., 2000 Martinez-Arias et al., 1988. Schwartz et al., 1995, DiNardo et al., 1988 Ingham et al., 1991, Bejsovec and Wieschaus, 1993]. c) Top: wingless expression pattern in an engrailed knock-out mutant embryo at stage 11 [Tabata et al., 1992]. The initial periodic pattern is disappearing, and gives rise to a non-segmented, embryonic lethal phenotype. Bottom: Non-segmented steady state of the Boolean model, obtained when wg, en or hh are kept off, or cell-to-cell signaling is disrupted [Albert and Othmer, 2003]. This steady state agrees with all experimental observations on $w g$, en, hh mutants [Tabata et al., 1992 DiNardo et al., 1988, Schwartz et al., 1995, Hidalgo and Ingham, 1990 Gallet et al., 2000]. Gene expression images obtained from http://www.fruitfly.org (a) and [Tabata et al., 1992] (b,c). 
during stages 9-11. Indeed, $w g$ and WG are expressed in the most posterior cell of each parasegment, while en, EN, $h h$ and $\mathrm{HH}$ are expressed in the most anterior cell of each parasegment, as is observed experimentally [Ingham 1998, Tabata et al., 1992], ptc is expressed in two stripes of cells, one stripe on each side of the en-expressing cells, the anterior one coinciding with the $w g$ stripe [Hidalgo and Ingham, 1990. Hooper and Scott, 1992]. $c i$ is expressed almost ubiquitously, with the exception of the cells expressing en [Eaton \& Kornberg 1990]. CIA is expressed in the neighbors of the HH-expressing cells, while CIR is expressed far from the HH-expressing cells [Aza-Blanc \& Kornberg 1999]. The model indicates that knockout mutations in en, wg, hh cause the non-segmented gene pattern shown on Fig. 2b, which agrees with experimental observations. Indeed, the $h h$ expression in en null embryos starts normally, but disappears before stage 10 [Tabata et al., 1992]. In $w g$ null embryos, en is initiated normally but fades away by stage 9, as observed by DiNardo et al. (1988), while $c i$ is ubiquitously expressed [Schwartz et al., 1995]. In $h h$ mutant embryos the $w g$ expression disappears by stage 10 [Hidalgo and Ingham, 1990], as does the expression of $p t c$, and there is no segmentation [Gallet et al., 2000]. On the other hand, ptc knockout mutations or overexpressed initial states lead to the broad-striped pattern of Fig. $2 \mathrm{c}^{2}$. Indeed, experimental results indicate broad $e n, w g$ and $h h$ stripes [Tabata et al., 1992 Gallet et al., 2000, Martinez-Arias et al., 1988] and Gallet et al. (2000) find that a new ectopic groove forms at the second en $-w g$ interface at the middle of the parasegment. Also, $c i$ is not expressed at this ectopic groove [Schwartz et al., 1995]. In heat-shock experiments the $w g$ and $p t c$ stripes expand anteriorly when $h h$ or $e n$ are ubiquitously induced [Gallet et al., 2000], while narrower $c i$ stripes emerge after a transient decay of $c i$ [Schwartz et al., 1995]. Intriguingly, the [Albert and Othmer, 2003] model finds that a knock-out mutation of $c i$ does not change the $e n, w g, h$ patterns but disrupts $p t c$ expression; experiments indicate that the segmental grooves are present and $w g$ is expressed until stage 11, but ptc expression decays [Gallet et al., 2000]. In summary, the simple synchronous Boolean model [Albert and Othmer, 2003] captures perfectly the wild type and mutant expression patterns of the segment polarity genes, and thus serves as a good starting point for a more realistic model that relaxes the assumption of synchronicity.

We focus our attention on a single parasegment of four cells, thus the total number of nodes we consider is $4 \times 13=52$. We use the same interaction topology and logical rules as the synchronous model Albert and Othmer, 2003, but instead of assuming that the states of all nodes are updated simultaneously, we update the state of each node individually (see Table11. To maintain the highest generality, we incorporate possible cell to cell variations in synthesis and decay processes. ${ }^{3}$

Throught the text, the notation " $w g_{1}^{t}$ " or " $w g_{1}(t)$ " represent the state of wingless mRNA in the first cell of the parasegment at time $t$. Similar notations apply for other mRNAs and proteins. There are 4 cells in each parasegment, and we adopted periodic boundary conditions, meaning that: node $_{4+1}=$ node $_{1}$ and node $_{1-1}=$ node $_{4}$. The wild type initial state corresponds to:

$$
w g_{4}^{0}=1, \quad e n_{1}^{0}=1, \quad h h_{1}^{0}=1, \quad p t c_{2,3,4}^{0}=1, \quad c i_{2,3,4}^{0}=1
$$

and the remaining nodes are zero. The asynchronous model represented in Table 1 exhibits the same steady states as the synchronous model developed in Albert and Othmer, 2003. Note that three of the four main steady states agree perfectly with experimentally observed states corresponding to wild type, en, wg or hh mutant and ptc mutant embryonic patterns [Tabata et al., 1992 DiNardo et al., 1988, Schwartz et al., 1995.

\footnotetext{
${ }^{2}$ The only difference between the $p t c$ mutant and heat-shock pattern is that the former does not express $p t c$ and PTC

${ }^{3}$ We follow the Albert and Othmer, 2003] model in assuming very short timescales for PTC-HH binding and SMO activation, and consequently in Figure 1 and in the regulatory rules we connect the CI posttranslational modifications to $\mathrm{HH}$ signaling. We have verified that this assumption can be relaxed without any qualitative changes in the results.
} 
Table 1: Regulatory functions governing the states of segment polarity gene products in the model . Each node is labeled by its biochemical symbol and subscripts signify cell number. The times $\tau^{j}$ signify the last time node $j$ was updated before $t$.

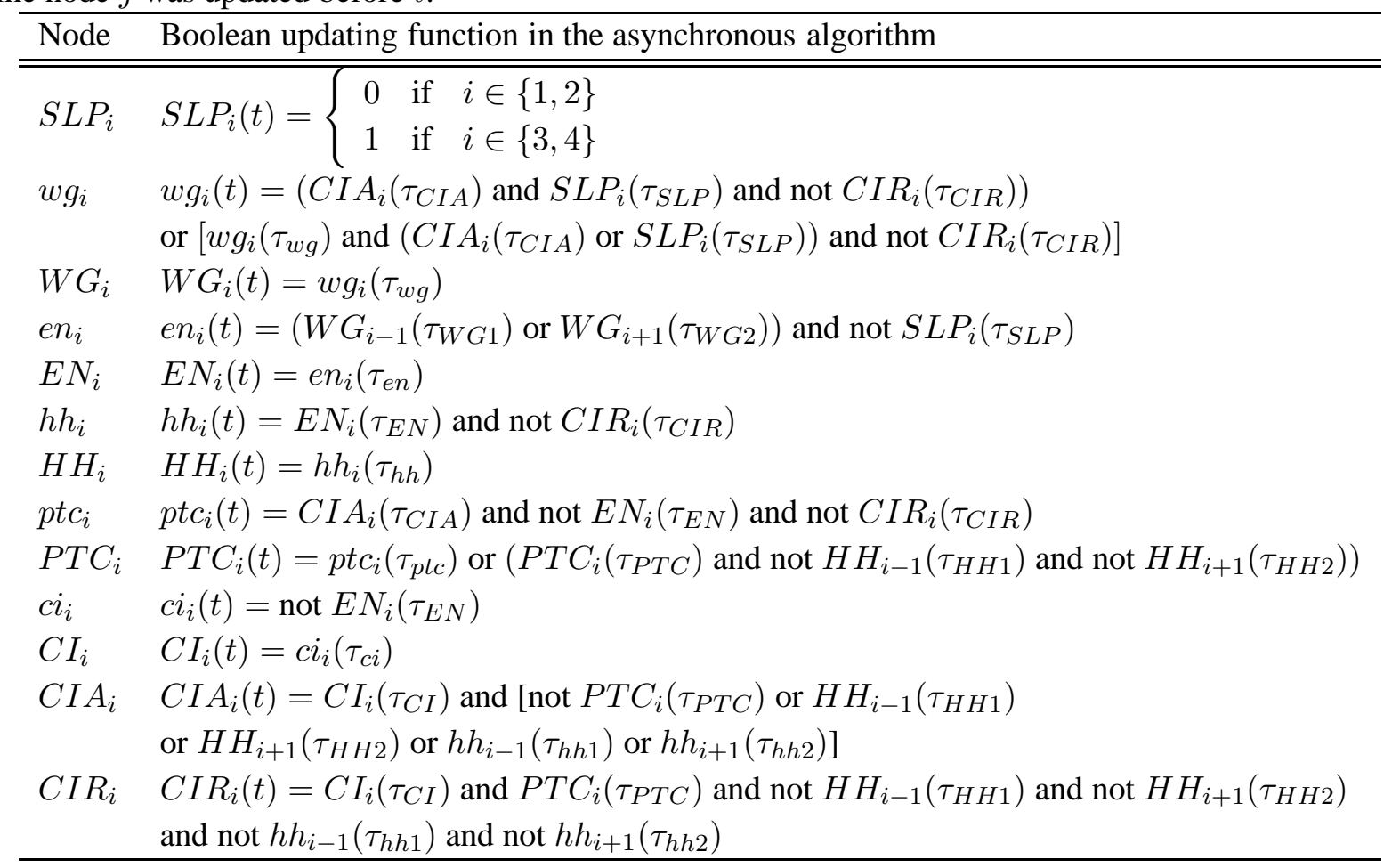

Hidalgo and Ingham, 1990, Gallet et al., 2000, Martinez-Arias et al., 1988 Bejsovec and Wieschaus, 1993. Ingham et al., 1991, Hooper and Scott, 1992, Wolpert et al., 1998]. A summary is presented in Table 2

\section{Randomly perturbed timescales}

As in the context of parallel computation systems, the fundamental difference between synchronous and asynchronous updates is at the level of task coordination and data communication among nodes in a network [Bertsekas and Tsitsiklis, 1989]. Synchronous algorithms are highly coordinated: at pre-determined instants, all the nodes "stop" and exchange the current information among themselves. For instance, suppose there are $N$ nodes, where each node $i$ "computes" the state of variable $x_{i}$, according to a function $f_{i}\left(x_{1}, x_{2}, \ldots, x_{N}\right)(i=1, \ldots, N)$. When all the $N$ nodes have finished phase $k$, they exchange their current states, $x_{i}^{k}$, and then proceed to phase $k+1$, that is

$$
x_{i}^{k+1}=f_{i}\left(x_{1}^{k}, x_{2}^{k}, \ldots, x_{N}^{k}\right) .
$$

Asynchronous algorithms, on the other hand, admit a greater flexibility at the level of process coordination. Each node is allowed to have its own "computation rate", that is, during any time interval $\left[t_{a}, t_{b}\right]$, node $i$ may be updated only once, while node $j$ may be updated $\ell>1$ times. In this case, communication delays between nodes may occur, and some possibly outdated information may be used: for instance, node $j$ uses the same value $x_{i}\left(t_{a}\right)$ throughout its $\ell$ updates in the interval $\left[t_{a}, t_{b}\right]$. However, an overall gain in efficiency in achieving the final result may be expected. For instance, in our example, the wild type steady state is reached in less than 4 steps with the asynchronous algorithms (see Sections 4 (5), while with the synchronous algorithm 6 steps are needed [Albert and Othmer, 2003. 
Table 2: Complete characterization of the model's steady states.

\begin{tabular}{ll}
\hline Steady state & Expressed nodes \\
\hline \hline wild type & $w g_{4}, W G_{4}, e n_{1}, E N_{1}, h h_{1}, H H_{1}$, \\
& $p t c_{2,4}, P T C_{2,3,4}, c i_{2,3,4}, C I_{2,3,4}, C I A_{2,4}, C I R_{3}$ \\
\hline broad stripes & $w g_{3,4}, W G_{3,4}, e n_{1,2}, E N_{1,2}, h h_{1,2}, H H_{1,2}$, \\
& $p t c_{3,4}, P T C_{3,4}, c i_{3,4}, C I_{3,4}, C I A_{3,4}$ \\
\hline no segmentation & $c i_{1,2,3,4}, C I_{1,2,3,4}, P T C_{1,2,3,4}, C I R_{1,2,3,4}$ \\
\hline wild type variant & $w g_{4}, W G_{4}, e n_{1}, E N_{1}, h h_{1}, H H_{1}$, \\
& $p t c_{2,4}, P T C_{1,2,3,4}, c i_{2,3,4}, C I_{2,3,4}, C I A_{2,4}, C I R_{3}$ \\
\hline ectopic & $w g_{3}, W G_{3}, e n_{2}, E N_{2}, h h_{2}, H H_{2}$, \\
& $p t c_{1,3}, P T C_{1,3,4}, c i_{1,3,4}, C I_{1,3,4}, C I A_{1,3}, C I R_{4}$ \\
\hline ectopic variant & $w g_{3}, W G_{3}, e n_{2}, E N_{2}, h h_{2}, H H_{2}$, \\
& $p t c_{1,3}, P T C_{1,2,3,4}, c i_{1,3,4}, C I_{1,3,4}, C I A_{1,3}, C I R_{4}$ \\
\hline
\end{tabular}

In general, we may say that node $i$ updates its state at times:

$$
T_{i}^{1}, T_{i}^{2}, \ldots, T_{i}^{k}, \ldots \quad k \in \mathbb{N}_{0},
$$

and the local variables, $x_{i}$, are updated according to:

$$
x_{i}\left[T_{i}^{k}\right]=f_{i}\left(x_{1}\left[\tau_{1 i}^{k}\right], \ldots, x_{N}\left[\tau_{N i}^{k}\right]\right),
$$

where $\tau_{j i}^{k}$ is defined as

$$
\tau_{j i}^{k}=\text { the latest available communication to node } i \text {, from node } j .
$$

There is usually a distinction between totally or partially asynchronous algorithms: the latter impose an updating constraint (every variable is updated at least once in any interval of a fixed length), while the former simply ensure that a variable is updated infinitely many times.

In a first numerical experiment we consider a totally asynchronous algorithm, with the highest degree of individual variability in each process' duration. The time unit of the synchronous model is randomly perturbed, so that the set of updating times for each node $i(1 \leq i \leq N)$ is of the form

$$
T_{i}^{k+1}=T_{i}^{k}+1+\varepsilon r_{i}^{k}, \quad k \in \mathbb{N},
$$

where $r_{i}^{k}$ are random numbers generated at each iteration, out of a uniform distribution in the interval $[-1,1]$. The value $\varepsilon \in[0,1)$ is the magnitude of the perturbation (the case $\varepsilon=0$ coincides with the synchronous algorithm). At any given time $t$, the next node(s) to be updated is(are) $j$ such that $T_{j}^{\ell}=\min _{i, k}\left\{T_{i}^{k} \geq t\right\}$, for some $\ell$. Since the duration of synthesis and decay processes is not known, through this algorithm one may randomly explore the space of all possible timescales and update orders, and derive the probability of different outcomes. The set of updating times $\left\{T_{i}^{k}, k \in \mathbb{N}_{0}\right\}$ may vary with each execution of the algorithm, so an element of stochasticity is naturally introduced.

Always starting from the wild type initial condition (1), this experiment was conducted over a wide range of perturbations $\left(10^{-12} \leq \varepsilon \leq 0.65\right)$, and 30000 trials were executed for each $\varepsilon$. The results (see Figure 3) show that all of the model's steady states may occur with a certain frequency: the wild type pattern with only $57 \%$, followed by the broad-striped pattern (24\%) observed in heat-shock experiments and ptc 


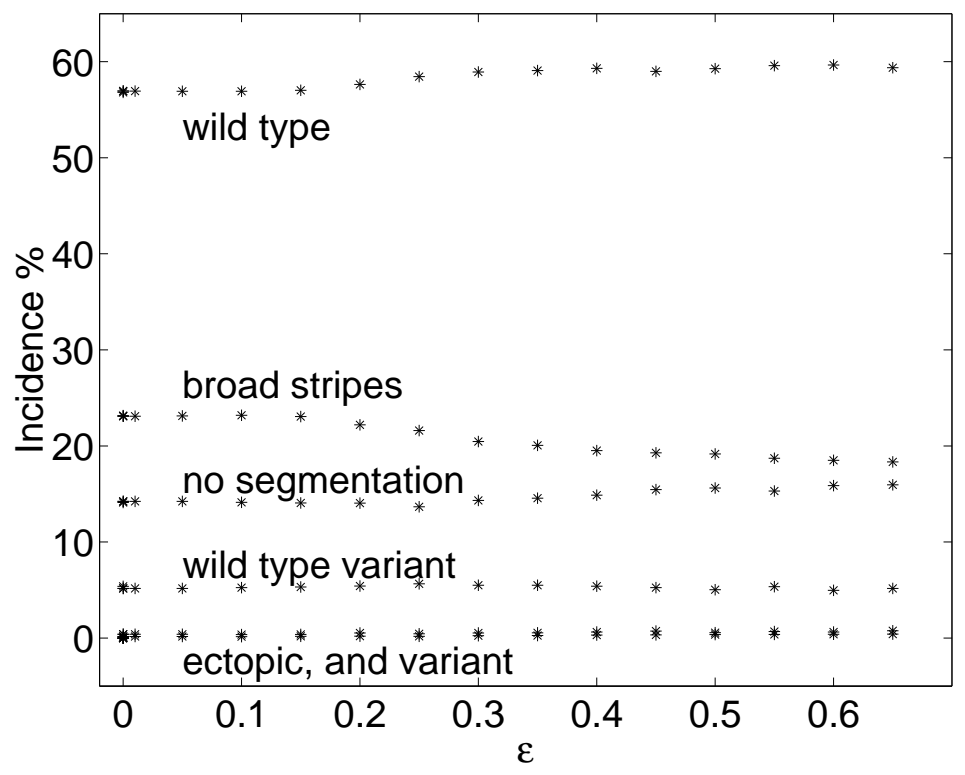

Figure 3: Fragility of the regulatory network. With the totally asynchronous algorithm the wild type initial state can lead to one of the six distinct steady states. Each $*$ corresponds to an $\varepsilon$ perturbation of the unit time-step. Note that the $\varepsilon \rightarrow 0$ limit does not give the same results as a synchronous update, demonstrating the fundamental difference between synchronous and asynchronous models.

mutants [Gallet et al., 2000] and by the pattern with no segmentation (15\%) observed in en, $h h$ or $w g$ mutants [Tabata et al., 1992], the latter two corresponding to embryonic lethal phenotypes [Gallet et al., 2000].

We observe that each of the steady state patterns occurs with a frequency which is independent of the value of $\varepsilon$, for $\varepsilon<0.15$. This may indicate that it is the order in which the protein and mRNA nodes are updated that determines the steady state pattern. In order to test this hypothesis, we designed a second experiment assuming that

(A1) Every node is updated exactly once during each unit time interval $(k, k+1](k=0,1,2, \ldots)$, according to a given order $\phi^{k}$.

This order $\phi^{k}$ is a permutation of $\{1, \ldots N\}$, chosen randomly (again out of a uniform distribution over the set of all $N$ ! possible permutations) at the beginning of the time unit $k$. Then we have

$$
T_{i}^{k}=N(k-1)+\phi^{k}(i), \quad k \in \mathbb{N}
$$

so that $\phi^{k}(j)<\phi^{k}(i)$ implies $T_{j}^{k}<T_{i}^{k}$, and node $j$ is updated before node $i$. The partially asynchronous algorithm leads to the same patterns, with incidence rates very similar to those observed with the totally asynchronous algorithm (see Table 3].

These results indicate the fragility of the wild type gene pattern with respect to changes in the timescales of synthesis and decay processes. While more than half of the random timescale combinations still lead to the expected outcome, a considerable percentage results in loss of the prepattern and an inviable final state.

\subsection{Imbalance between CIA and CIR}

Further analysis shows that the divergence from wild type can be attributed to an imbalance between the two opposing Cubitus Interruptus transcription factors (CIA, CIR) in the posterior half of the parasegment. 
Table 3: The frequencies of the six steady states observed in the partially asynchronous model confirm those observed for the totally asynchronous model. The frequencies are computed from 30000 executions.

\begin{tabular}{ll}
\hline Steady State & Incidence \\
\hline \hline wild type & $56 \%$ \\
broad stripes & $24 \%$ \\
no segmentation & $15 \%$ \\
wild type variant & $4.2 \%$ \\
ectopic & $0.98 \%$ \\
ectopic variant & $0.68 \%$ \\
\hline
\end{tabular}

Indeed, the expression of CIA and CIR in both the broad stripes and the no segmentation patterns is clearly distinct from that in the wild type pattern. In the next set of numerical experiments, we explore the effects of CIA/CIR expression in the formation of the final pattern.

In wild type, the two Cubitus Interruptus proteins, CIA and CIR, are expressed in different cells of the posterior part of the parasegments, namely,

$$
\begin{array}{ll}
C I A_{3}=0, & C I A_{4}=1, \\
C I R_{3}=1, & C I R_{4}=0,
\end{array}
$$

and the maintenance of these complementary ON/OFF states is essential in the wild type pattern. To investigate the effect of an imbalance between the two Cubitus Interruptus proteins, we considered two disruptive cases: the (transient) overexpression of CIR, or the (transient) overexpression of CIA and absence of CIR in both posterior cells.

More precisely, in the totally asynchronous algorithm (choosing $\varepsilon=0.1$ ), we transiently imposed an expression pattern for the Cubitus proteins as follows:

(a) $C I A_{3,4}^{t}=1$ and $C I R_{3,4}^{t}=0$, for $t \in[3,3+\tau]$;

(b) $C I R_{3,4}^{t}=1$, for $t \in[3,3+\tau]$,

where $\tau$ is the duration of the transient. The overexpression starts after three unit time steps. The duration of the transient was:

$$
\tau \in\{0,0.3,0.75,1.5,2.75,3\}
$$

so when $\tau=0$ the results of the general totally asynchronous algorithm are recovered.

Our results show that even a small transient imbalance between CIA and CIR causes a clear bias towards a mutant state: the broad stripes mutant in case (a), or the no segmentation mutant in case (b). Thus any perturbation that leads to such an imbalance has as severe effects as a mutation in ptc (causing the broad striped pattern) or either of $e n, w g$ or $h h$ (causing the nonsegmented pattern).

These numerical experiments also open the way to many other questions: are there particular sequences that lead to a given steady state? How is the evolution from the initial to steady state? How robust is the asynchronous model with respect to initial conditions? 

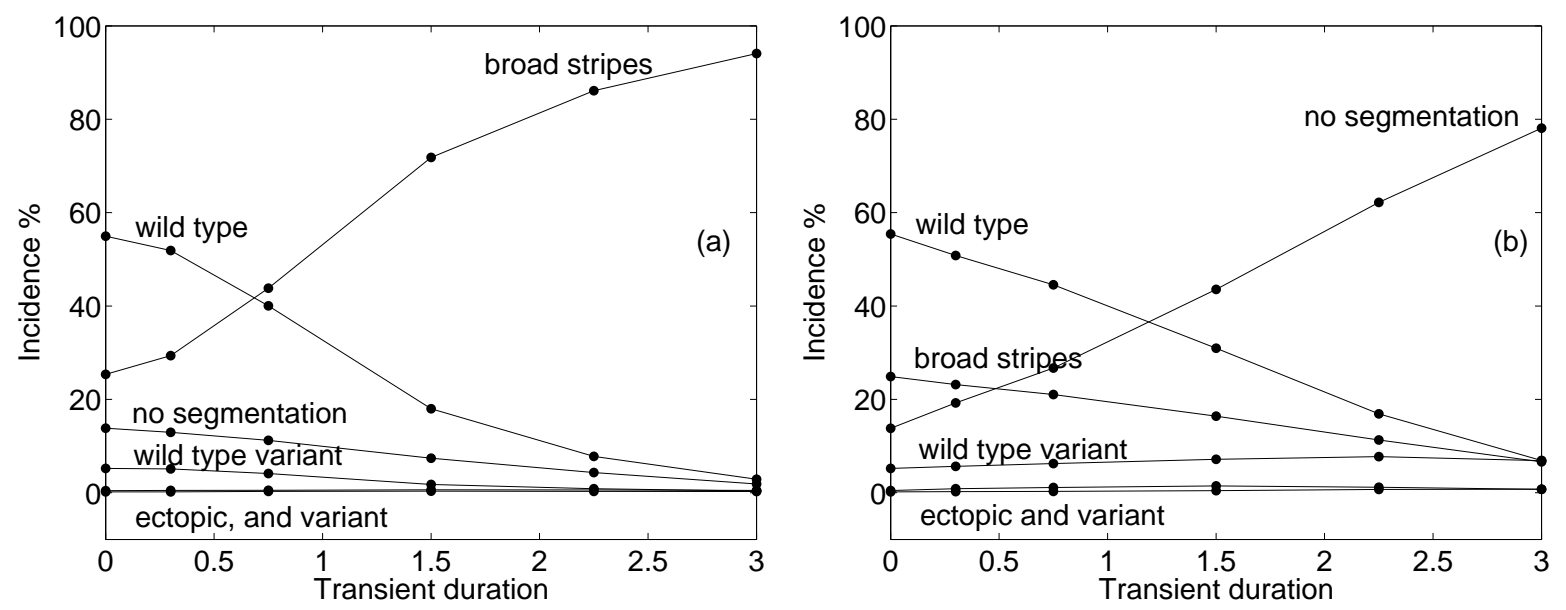

Figure 4: Bias towards mutant states. The $x$-axis represents the duration of the transient, $\tau$ (in unit time steps). The incidence probabilities were computed over 20000 trials. (a) The case $C I A_{3,4}^{t}=1$ and $C I R_{3,4}^{t}=0$ leads to the broad striped pattern. (b) The case $C I R_{3,4}^{t}=1$ leads to the no segmentation pattern.

\section{Timescale separation uncovers robustness of the model}

In both of the previous algorithms we assumed no bias towards a preferred protein/mRNA updating sequence and, as a result, an unrealistic divergence from the wild type pattern is observed, with high incidence of inviable states. Based on the fact that post-translational processes such as protein conformational changes or complex formation usually have shorter durations than transcription, translation or mRNA decay, we introduce a distinct timescale separation by choosing to update proteins first and mRNAs later. This leads to a model which is very robust, in the sense that the wild type pattern occurs with a frequency of $87.5 \%$ and only one other steady state is observed, the broad striped pattern, with a frequency of $12.5 \%$. We completely characterize this model by theoretically showing that only two of the six steady states are possible (and occur with well determined frequencies), and identifying the order of updates that leads to divergence from wild type. We also show that the wild type state is really an attractor for the system, while the pathway to the broad stripes state may show oscillatory cycles.

Assuming that

(A2) All the proteins are updated before all the genes,

the $k$-th iteration of the two-timescale algorithm proceeds as follows:

(A3) At the begining of the $k$-th time unit, generate a random permutation, $\phi_{\text {Prot }}^{k}$ of $\{1, \ldots L\}$, and a random permutation, $\phi_{m R N A}^{k}$ of $\{L+1, \ldots N\}$ (using a uniform d istribution over, respectively, the sets of $L$ ! and $(N-L+1)$ ! possible permutations). Then the $N$ nodes are updated in the order given by $\phi^{k}=\left(\phi_{\text {Prot }}^{k}, \phi_{m R N A}^{k}\right)$, according to (2), with

$$
\tau_{j i}^{k}=\left\{\begin{array}{cc}
T_{j}^{k-1}, & \phi^{k}(j) \leq \phi^{k}(i) \\
T_{j}^{k}, & \phi^{k}(j)>\phi^{k}(i) .
\end{array}\right.
$$

As an example, suppose that

$$
N=5, \quad L=3, \quad \phi_{\text {Prot }}^{1}=\{2,1,3\}, \quad \phi_{m R N A}^{1}=\{5,4\} .
$$


Table 4: Regulatory functions governing the states of segment polarity gene products in the two-timescale asynchronous algorithm. Each node is labeled by its biochemical symbol, subscripts signify cell number and superscripts signify timestep. Although the updating time of each node varies, each function can be written by using the states of effector nodes at the previous or current timesteps. The individual times $t_{1} \ldots t_{10}$ can take the values $\{t-1, t\}$.

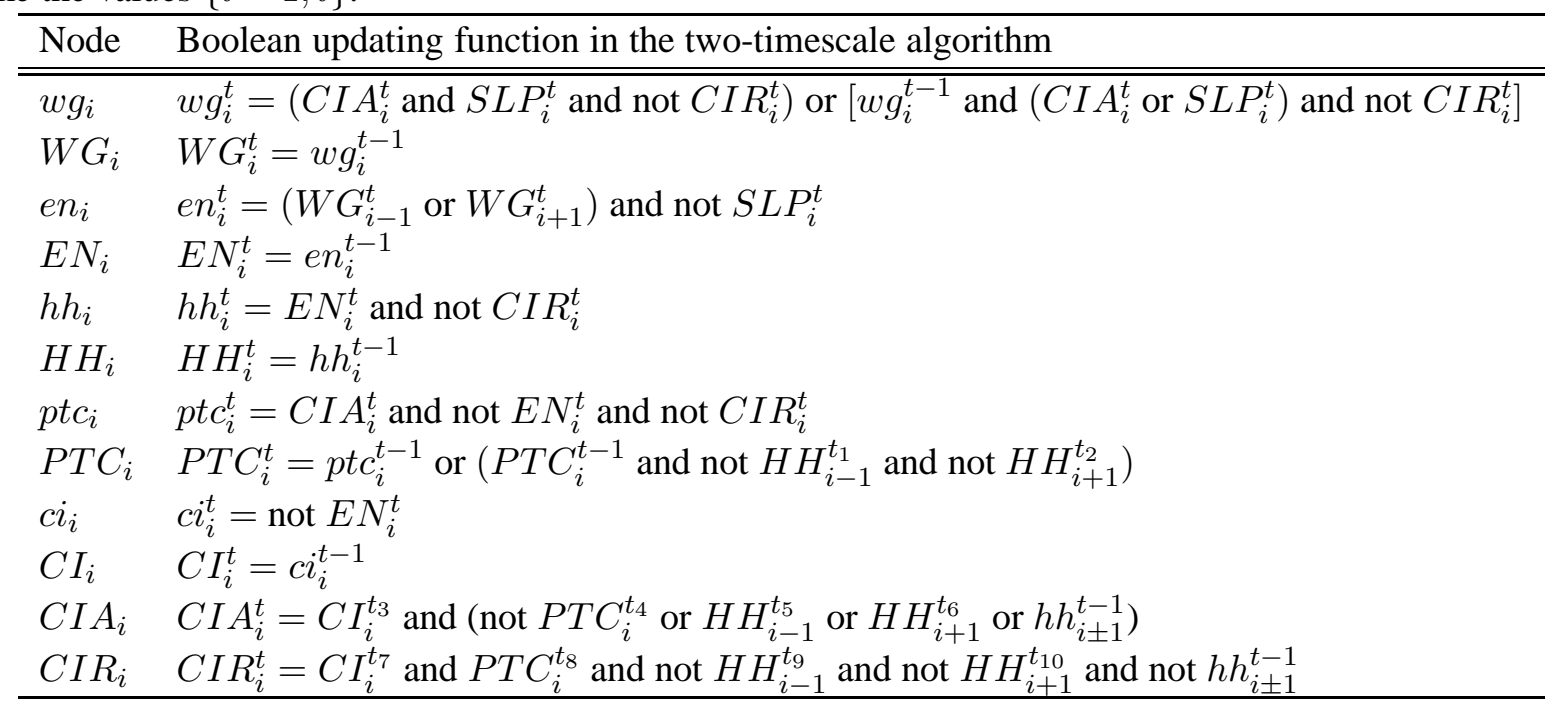

Then, $\phi^{1}=\{2,1,3,5,4\}$, and $T_{1}^{1}=2, T_{2}^{1}=1, T_{3}^{1}=3, T_{4}^{1}=5, T_{5}^{1}=4$. The nodes are updated as follows (for simplicity of notation, we will write $x_{i}^{k}:=x_{i}\left[T_{i}^{k}\right]$ ):

$$
\begin{aligned}
x_{2}^{1} & =f_{2}\left(x_{1}^{0}, x_{2}^{0}, x_{3}^{0}, x_{4}^{0}, x_{5}^{0}\right), \\
x_{1}^{1} & =f_{1}\left(x_{1}^{0}, x_{2}^{1}, x_{3}^{0}, x_{4}^{0}, x_{5}^{0}\right), \\
x_{3}^{1} & =f_{3}\left(x_{1}^{1}, x_{2}^{1}, x_{3}^{0}, x_{4}^{0}, x_{5}^{0}\right), \\
x_{5}^{1} & =f_{5}\left(x_{1}^{1}, x_{2}^{1}, x_{3}^{1}, x_{4}^{0}, x_{5}^{0}\right), \\
x_{4}^{1} & =f_{4}\left(x_{1}^{1}, x_{2}^{1}, x_{3}^{1}, x_{4}^{0}, x_{5}^{1}\right) .
\end{aligned}
$$

Some general inferences about the updating rules can be made. For example, the translation process only depends on the presence of the transcript, which is decided in the previous time unit, thus $\operatorname{Prot}^{t}=m R N A^{t-1}$. The beginning of a transcription process depends on the presence of transcription factors, and since mRNAs are updated after proteins, $m R N A^{t}=$ Prot $^{t}$. The outcome of post-translational processes depends on the order of updates, for example the rule for a binding process will be Complex ${ }^{t}=$ Prot $_{1}^{t_{1}}$ and Prot $_{2}^{t_{2}}$, where $t_{1}$ and $t_{2}$ can be either $t-1$ or $t$ (see Table 4).

\subsection{Two steady states}

The trajectory of the system is thus defined by a sequence of permutations (obtained as described in A3) and the corresponding sequence of states:

$$
\left\{\phi^{k}\right\},\left\{x^{k}\right\} \text { for } k=0,1,2, \ldots
$$

We will show that for pre-patterns that satisfy $w g_{4}^{0}=1, c i_{1}^{0}=0$ and $p t c_{1}^{0}=0$ (which include the pattern observed in the wild type at stage 8), the only possible steady states for system (3) are the wild type pattern experimentally observed at stages 9-11, and a mutant with broad $w g$ stripes. We assume that all the proteins are absent initially (at $T=0$ ), and that the which sloppy pair gene is maintained at a constant value: 
$S L P_{1,2}=0$ and $S L P_{3,4}=1$ This pattern for SLP is responsible for permanent absence (or expression) of some of the segment polarity genes, and corresponding proteins, in certain cells of the parasegment. By direct inspection of the model, it follows that

$$
\begin{gathered}
w g_{1,2}^{0}=0, \Rightarrow w g_{1,2}^{T}=0, \quad W G_{1,2}^{T}=0, \text { for } T \geq 0 \\
e n_{3,4}^{T}=0, \quad E N_{3,4}^{T}=0, \text { for } T \geq 0 \\
h h_{3,4}^{T}=0, \quad H H_{3,4}^{T}=0, \text { for } T \geq 0
\end{gathered}
$$

and

$$
\begin{gathered}
c i_{3,4}^{0}=1, \Rightarrow c i_{3,4}^{T}=1, \text { for } T \geq 0, \text { and } C I_{3,4}^{T}=1, \text { for } T \geq 1 \\
c i_{3,4}^{0}=0, \Rightarrow c i_{3,4}^{T}=1, \text { for } T \geq 1, \text { and } C I_{3,4}^{T}=1, \text { for } T \geq 2 .
\end{gathered}
$$

The next statement reflects the fact that the effect of $w g_{4}$ activating $e n_{1}$ propagates to inhibit $c i_{1}$ which then eliminates all forms of CI from the first cell.

Fact 1. Assume that $w g_{4}^{0}=1, c i_{1}^{0}=0$ and $p t c_{1}^{0}=0$. For any $T \geq 0$, if $w g_{4}^{t}=1$ for all $0 \leq t \leq T$, then $C I_{1}^{t}=0$ for all $3 \leq t \leq T+3$ and $C I R_{1}^{t}=0$ for all $0 \leq t \leq T+3$.

Proposition 4.1. Assume $w g_{4}^{0}=1, c i_{1}^{0}=0$ and $p t c_{1}^{0}=0$. Under assumptions A1-A2, $w g_{4}^{T}=1$, for all $T \geq 0$.

Proof. We will argue by contradiction. Suppose that there do exist times $t \geq 1$ with $w g_{4}^{t}=0$, and let $T$ be the minimum of such times, that is,

$$
w g_{4}^{T}=0 \text { and } w g_{4}^{t}=1, \text { for all } 0 \leq t<T .
$$

¿From the model's equations, together with assumptions A1-A2:

$$
\begin{aligned}
W G_{4}^{t} & =w g_{4}^{t-1}, \\
w g_{4}^{t} & =\left(C I A_{4}^{t} \text { and not } C I R_{4}^{t}\right) \text { or }\left(w g_{4}^{t-1} \text { and not } C I R_{4}^{t}\right),
\end{aligned}
$$

for all $t \geq 1$. So, it follows that

$$
\begin{aligned}
& W G_{4}^{t}=1, \quad \text { for all } 0 \leq t \leq T, \\
& C I R_{4}^{t}=0, \quad \text { for all } 0 \leq t<T, \quad \text { and } C I R_{4}^{T}=1 .
\end{aligned}
$$

Now, from Fact 1 it also follows that

$$
C I R_{1}^{t}=0 \text { for all } 0 \leq t \leq T+2 .
$$

The equation for $\mathrm{CIR}_{4}$ is:

$$
C I R_{4}^{t}=C I_{4}^{t_{d}} \text { and not }\left[\text { not } P T C_{4}^{t_{a}} \text { or } H H_{3}^{t_{c}} \text { or } H H_{1}^{t_{b}} \text { or } h h_{3}^{t-1} \text { or } h h_{1}^{t-1}\right. \text { ] }
$$

(where $t_{a}, \ldots, t_{d} \in\{t, t-1\}$ depend on the permutation $\phi^{t}$ ). Recall also that

$$
\begin{aligned}
h h_{1}^{t} & =E N_{1}^{t} \text { and not } C I R_{1}^{t} \\
E N_{1}^{t} & =e n_{1}^{t-1} \\
e n_{1}^{t-1} & =W G_{4}^{t-1} \text { or } W G_{2}^{t-1}
\end{aligned}
$$


From (12):

$$
C I R_{4}^{T}=1 \Rightarrow h h_{1}^{T-1}=0
$$

and then from (11) and (14):

$$
h h_{1}^{T-1}=0 \Rightarrow E N_{1}^{T-1}=0 .
$$

Now by equations 15 16:

$$
E N_{1}^{T-1}=0, \Rightarrow e n_{1}^{T-2}=0 \Rightarrow W G_{4}^{T-2}=0 \text { and } W G_{2}^{T-2}=0,
$$

which contradicts equation (9). Thus, it must be that $w g_{4}^{T}=1$ for all times $T$, as we wanted to show.

The following are now immediate conclusions from the model.

Corollary 4.2. $C I R_{4}^{T}=0$ for all $T \geq 0, e n_{1}^{T}=1$ and $W G_{4}^{T}=1$ for all $T \geq 1 . E N_{1}^{T}=1, c i_{1}^{T}=0$ and $h h_{1}^{T}=1$ for all $T \geq 2$. $C I_{1}^{T}=0$ and $H H_{1}^{T}=1$ for all $T \geq 3$. And finally, $C I A_{1}^{T}=C I R_{1}^{T}=0$ for all $T \geq 4$.

Corollary 4.3. $p t c_{1}^{T}=0$ and $P T C_{1}^{T}=0$ for all $T \geq 0$, and $C I R_{2}^{T}=0$ for all $T \geq 3$.

In conclusion, from Proposition 4.1 it is clear that neither the no segmentation nor the two ectopic patterns are steady states of the system (3) under assumptions A1-A2, because all of these states imply $w g_{4}=0$. In addition, Corollary 4.3 shows that the wild type variant, where PTC is ubiquitous, cannot be a steady state. Also, any of the states with $w g_{1,2}=1$ is immediately prevented by the initial condition (4). This leaves only the "regular" wild type or the mutant with broad $w g$ stripes.

\subsection{Divergence from wild type}

Under assumptions A1-A2, divergence from the wild type pattern occurs if and only if the first permutation (in particular $\phi_{\text {Prot }}^{1}$ ) is of a particular form. Thus, convergence (or divergence) to the wild type pattern is decided at the first iterate $(T=1)$.

Recall that the wild type pattern requires wingless not to be expressed in the third cell $\left(w g_{3}=0\right)$. The next Fact (proved in the Appendix) essentially says that a stable $w g_{3}=0$ induces the absence of both engrailed, hedgehog in the second cell, as well as the absence of $\mathrm{CIA}_{3}$, and maintains the expression of $\mathrm{PTC}_{3}$.

Fact 2. Assume $p t c_{3}^{0}=1$ and $e n_{2}^{0}=0$.

(a) Let $T \geq 1$. If $w g_{3}^{t}=0$ for all $0 \leq t \leq T$, then

$$
\begin{aligned}
& e n_{2}^{t}=0, E N_{2}^{t}=0, \quad 0 \leq t \leq T+2, \\
& h h_{2}^{t}=0, \quad 1 \leq t \leq T+2, \\
& H H_{2}^{t}=0, \quad 1 \leq t \leq T+3, \\
& P T C_{3}^{t}=1, \quad 1 \leq t \leq T+3, \text { and } \\
& C I A_{3}^{t}=0, C I R_{3}^{t}=1, \quad 2 \leq t \leq T+3 \text {. }
\end{aligned}
$$

(b) Furthermore, if $c i_{3}^{0}=0$, then also $C I A_{3}^{1}=0$ and part (a) holds for any $T \geq 0$. 
With the help of this Fact, we establish that $w g_{3}$ may become expressed only at the first iterate or else it is never expressed. Thus the two timescale model provides a strong natural restriction on the formation of an inviable state: if $w g_{3}^{1}=0$, then $w g_{3}^{T}=0$ for all $T \geq 0$, implying that such trajectories will never converge to the broad striped pattern.

Proposition 4.4. Assume that the initial condition satisfies $w g_{3}^{0}=0, p t c_{3}^{0}=1, h h_{2,4}^{0}=0$, and $c i_{3}^{0}=1$. Then $w g_{3}^{T_{1}}=1$ and $w g_{3}^{T}=0$ for all $0 \leq T<T_{1}$, only if $T_{1}=1$.

Proof. To obtain $w g_{3}^{T}=1$ with $w g_{3}^{T-1}=0$ it is necessary that

$$
w g_{3}^{T}=C I A_{3}^{T} \text { and not } C I R_{3}^{T} \Rightarrow C I A_{3}^{T}=1 \text { and } C I R_{3}^{T}=0 .
$$

But, if $w g_{3}^{T}=0$ for $T=1$, then, by Fact 2 the activator $C I A_{3}$ is zero for $T=2,3,4$. Then (by induction on $T$ ) expression of $w g_{3}$ is prevented at any later time.

In addition, it is possible to completely characterize the updating permutation $\left(\phi^{1}\right)$ that leads to $w g_{3}^{1}=1$ and, as a consequence, exactly compute the probability of divergence (hence convergence) to the wild type steady state (Section 4.3).

Proposition 4.5. Assume that assumptionts A1 and A2 hold. Assume that the initial condition satisfies $w g_{3}^{0}=0, p t c_{3}^{0}=1$ and $h h_{2,4}^{0}=0$.

(a) If $c i_{3}^{0}=0$, then $w g_{3}^{1}=0$.

(b) If $c i_{3}^{0}=1$, then $w g_{3}^{1}=1$ if and only if the permutation $\phi^{1}$ satisfies the following sequence among the proteins $C I, C I A, C I R$ and $P T C$ :

$$
\begin{array}{cccccc}
C I R_{3} & C I_{3} & & C I A_{3} & & \mathrm{PTC}_{3}, \\
& & & & & \\
C I_{3} & C I R_{3} & C I A_{3} & & P T C_{3}, \\
C I_{3} & & C I A_{3} & C I R_{3} & \mathrm{PTC}_{3},
\end{array}
$$

while the other proteins may appear in any of the remaining slots.

Proof. Part (a) follows immediately from Fact 2 (b). To prove part (b), we start by noticing that, because $S L P_{3}=1$ and $w g_{3}^{0}=0$,

$$
w g_{3}^{1}=C I A_{3}^{1} \text { and not } C I R_{3}^{1},
$$

so that

$$
w g_{3}^{1}=1 \quad \Leftrightarrow \quad C I A_{3}^{1}=1 \text { and } C I R_{3}^{1}=0 .
$$

Following assumptions A1-A2, the model's equations for $C I A_{3}^{1}$ and $C I R_{3}^{1}$ are given by:

$$
\begin{aligned}
& C I A_{3}^{1}=C I_{3}^{t_{a}} \text { and }\left[\operatorname{not} P T C_{3}^{t_{b}} \text { or } H H_{2}^{t_{c}} \text { or } H H_{4}^{t_{d}} \text { or } h h_{2}^{0} \text { or } h h_{4}^{0}\right], \\
& C I R_{3}^{1}=C I_{3}^{s_{a}} \text { and not }\left[\operatorname{not} P T C_{3}^{s_{b}} \text { or } H H_{2}^{s_{c}} \text { or } H H_{4}^{s_{d}} \text { or } h h_{2}^{0} \text { or } h h_{4}^{0}\right],
\end{aligned}
$$

where $t_{a}, \ldots, s_{a} \in\{0,1\}$ and depend on the permutation $\phi^{1}$. These expressions may be simplified by observing that: (a) $h h_{2,4}^{0}=0$, and thus also (b) $H H_{2,4}^{0,1}=0$. Therefore,

$$
\begin{aligned}
& C I A_{3}^{1}=C I_{3}^{t_{a}} \text { and not } P T C_{3}^{t_{b}}, \\
& C I R_{3}^{1}=C I_{3}^{s_{a}} \text { and } P^{1} C_{3}^{s_{b}},
\end{aligned}
$$

The values for $C I_{3}^{0,1}$ and $P T C_{3}^{0,1}$ are determined by: 
1. $C I_{3}^{0}=0$ and $C I_{3}^{1}=c i_{3}^{0}=1$,

2. $P T C_{3}^{0}=0$ and $P T C_{3}^{1}=p t c_{3}^{0}$ or $[\cdots]=1$, since $p t c_{3}^{0}=1$,

and recall that both $C I A_{i}^{0}=0$ and $C I R_{i}^{0}=0$. Therefore, it is necessary that $C I_{3}$ is updated before $C I A_{3}$ and $P T C_{3}$ is updated after $C I A_{3}$, because otherwise $C I A_{3}^{1}=0$. Finally, $C I R_{3}$ must be updated before $P T C_{3}$, because otherwise $C I R_{3}^{1}=1$. In other words:

$$
t_{a}=1, t_{b}=0, s_{a} \in\{0,1\}, s_{b}=0 .
$$

It is easy to see that any of the sequences (17) is also sufficient to obtain $w g_{3}^{1}=1$.

Finally, we will show that whenever $w g_{3}$ becomes expressed at time $T=1$, it is afterwards periodically expressed, every third step. Such trajectories cannot converge to the wild type pattern. In other words, initial permutations of the form (17) are not included in the basin of attraction of the wild type pattern.

Proposition 4.6. Assume $w g_{4}^{0}=1, c i_{1}^{0}=0$ and $p t c_{1}^{0}=0$. For any $T \geq 1$, if $w g_{3}^{T}=1$, then $w g_{3}^{T+3}=1$.

Proof. Recall that, by Proposition 4.1 $w g_{4}^{t}=1$ for all $t \geq 0$. By Corollary 4.3 CI $R_{2}^{t}=0$ for all $t \geq 3$.

Now, pick any $T \geq 1$ and assume that $w g_{3}^{T}=1$. Then

$$
W G_{3}^{T+1}=1 \Rightarrow e n_{2}^{T+1}=1 \Rightarrow E N_{2}^{T+2}=1 \Rightarrow h h_{2}^{T+2}=w g_{3}^{T}=1,
$$

where the last implication follows from Fact 4 Then (using either (7) or (8))

$$
\begin{aligned}
& C I A_{3}^{T+3}=\operatorname{not} P T C_{3}^{t_{a}} \text { or } H H_{2}^{t_{b}} \text { or } h h_{2}^{T+2}, \\
& C I R_{3}^{T+3}=\operatorname{PTC}_{3}^{s_{a}} \text { and not } H H_{2}^{s_{b}} \text { and not } h h_{2}^{T+2},
\end{aligned}
$$

(where $t_{a}, t_{b}, s_{a}, s_{b} \in\{T+2, T+3\}$, and depend on the permutation $\phi^{T+3}$ ). So $h h_{2}^{T+2}=1$ implies

$$
C I A_{3}^{T+3}=1 \text { and } C I R_{3}^{T+3}=0,
$$

and therefore $w g_{3}^{T+3}=1$, as we wanted to show.

Whenever $w g_{3}$ is not expressed, some other nodes also stabilize, after the appropriate number of iterations. These are summarized next.

Corollary 4.7. Assume that $w g_{3}^{t}=0$ for all $t \geq 0$. Then $W G_{3}^{t}=0$, ent $n_{2}^{t}=0$ for all $t \geq 1 . E N_{2}^{t}=0$, $h h_{2}^{t}=0$ and $c i_{2}=1$ for all $t \geq 2$. Finally, $H H_{2}^{t}=0$ and $C I_{2}=1$ for all $t \geq 3$.

\subsection{Probability of convergence to wild type}

The wild type pattern is in fact an attractor for the asynchronous model: every trajectory which is not of the form (17) converges to the wild type pattern (see Appendix B). The probability that this happens is therefore determined by counting all the possible states of the form (17):

$$
\operatorname{Prob}(\text { wild type })=1-\frac{\# \text { permutations as in }(17)}{\text { Total \# permutations }}
$$

Let $L$ be the number of protein nodes to be updated at each iterate (there are 9 proteins in each of the four cells so $L=36$ ). Out of the $L$ proteins, only 4 need to satisfy one of particular sequences (17) in their 
relative positions. So let $M_{L}$ be the number of possible permutations satisfying any of the sequences (17). Then

$$
\operatorname{Prob}(\text { wild type })=1-\frac{M_{L}(L-4) !}{L !} .
$$

The next Proposition is proved in the Appendix and shows that, in fact, this is a constant number, independent of $L$. That is,

$$
\text { Prob(wild type) }=0.875 \text {. }
$$

Proposition 4.8. For any $L \geq 4$,

$$
M_{L}=\frac{3 !}{2} \sum_{P=4}^{L}\left(\begin{array}{c}
P-1 \\
3
\end{array}\right)=\frac{1}{2} \sum_{j=1}^{L-3} j(j+1)(j+2)
$$

and

$$
\frac{M_{L}(L-4) !}{L !}=\frac{1}{8}
$$

\section{A Markov chain process}

As a Boolean model, there are only a finite set, say $\mathcal{S}$, of distinct states (in the total state space $\{0,1\}^{N}$ ) reachable by the system. Starting from any state $\mathcal{S}_{a} \in \mathcal{S}$, each permutation $\phi$ of $\{1, \ldots, N\}$ takes the system to some other state $\mathcal{S}_{b} \in \mathcal{S}$. It is possible to theoretically identify all the distinct intermediate and final states of the system as well as all the possible transitions after one iteration. Thus the asynchronous algorithm consisting of the $N$ node functions (2) together with assumptions (A1), (A2) and (A3) may be characterized as a Markov chain process, by identifying the $d$ distinct states

$$
\mathcal{S}=\left\{\mathcal{S}_{1}, \mathcal{S}_{2}, \ldots, \mathcal{S}_{d}\right\}
$$

and the $d \times d$ transition matrix $P$, where

$$
P_{i j}=\text { probability of a transition from state } \mathcal{S}_{i} \text { to state } \mathcal{S}_{j} .
$$

The probabilities $P_{i j}$ are simply the fraction of the total number of permutations that (in one iterate) transform the state $\mathcal{S}_{i}$ into state $\mathcal{S}_{j}$. The matrix $P$ is a stochastic matrix, since all its rows add up to 1 . A state $\mathcal{S}_{a}$ with the property that all permutations leave the state unchanged (that is, $P_{a a}=1$ and $P_{a j}=0$ for $j \neq 0$ ) is called an absorption state of the Markov chain, and it is also a steady-state of system (2). In the asynchronous model there are only two absorption states, corresponding to the wild type and broad wingless stripe mutant patterns, as described above. Isolating the two rows and columns that correspond to these absorption states, the transition matrix may be partitioned as

$$
P=\left[\begin{array}{ll}
P_{a} & 0 \\
R_{a} & \bar{P}
\end{array}\right]
$$

where $\bar{P}$ is of size $(d-2) \times(d-2)$. It is a well know result(see any standard book on probability theory, for instance [Feller, 1970]) that $I-\bar{P}$ is an invertible matrix, and

$$
\left[\begin{array}{c}
\bar{T}_{1} \\
\bar{T}_{2} \\
\vdots \\
\bar{T}_{d-2}
\end{array}\right]=(I-\bar{P})^{-1}\left[\begin{array}{c}
1 \\
1 \\
\vdots \\
1
\end{array}\right]
$$


or $\bar{T}_{i}=1+\sum_{j=1}^{d-2} p_{i j} \bar{T}_{j}$. The values $\bar{T}_{i}$ provide an estimated time for absorption when the chain starts from state $\mathcal{S}_{i}$ (if $a$ is an absorption state, then $\bar{T}_{a}=0$ ). In Figure [5] a schematic diagram of transitions is shown, together with probabilities and estimated times for absorption. This diagram was obtained from a simulation starting with the initial wild type pattern (observed at stage 8 of the embrionic development), and following the assumptions (A1)-(A3), as well as the additional

(A4) Each protein or gene is updated simultaneously in the four cells,

meaning that there is no cell-to-cell variation in the duration of molecular processes. In this case, the total number of possible permutations is a manageable $7 ! \times 5 !=604800$ :

$$
\begin{aligned}
\Phi=\left\{\phi=\left(\phi_{\text {Prot }}, \phi_{m R N A}\right): \quad\right. & \phi_{\text {Prot }} \text { is a permutation of }\{1,2,3,4,5,6,7\}, \\
& \left.\phi_{\text {mRNA }} \text { is a permutation of }\{1,2,3,4,5\}\right\}
\end{aligned}
$$

The total number of distinct states of the Markov chain (under assumptions (A1)-(A4)) is $d=48$. The transition probabilities matrix was computed exactly, by counting all the $7 ! \times 5$ ! transitions from each of the 48 states.

It is clear from this diagram that the decision between convergence to the wild type or the mutant patterns is indeed decided at the first iteration, in agreement with Propositions 4.1 and 4.4 Furthermore, the diagram shows the possibility of periodic oscillations (of period at least three) in the mutant branch (see also Proposition 4.6. Although in practice the probability of a limit cycle is very small, this prevents (theoretical) convergence to the mutant state, and considerably increases the absorption times to the mutant state. The robustness of the two timescale model is illustrated by the fast convergence to the wild type pattern (expected time to convergence is 4 steps), contrasted with the long and oscillation-strewn path toward the broad striped pattern (expected time to convergence is 15 steps).

\section{Identifying minimal pre-patterns}

A necessary condition for convergence to the wild type is that $p t c_{3}^{0}=1$. Otherwise the trajectory immediately fails to enter the basin of attraction of the will type state:

Fact 3. Assume $p t c_{3}^{0}=0$. Then $w g_{3}^{T}=1$, for some $T \in\{1,2,3\}$.

Proof. Note that $p t c_{3}^{0}=0$ implies $P T C_{3}^{1}=0$. Using (5) and (7), (8), the equations for $p t c_{3}$ and $C I A_{3}$ simplify to

$$
\begin{aligned}
C I A_{3}^{t} & =\operatorname{not} C I R_{3}^{t}=\operatorname{not} P T C_{3}^{t_{a}} \text { or } H H_{2}^{t_{b}} \text { or } h h_{2}^{t-1}, \quad t \geq \tau \\
p t c_{3}^{t} & =C I A_{3}^{t} \text { and not } C I R_{3}^{t}=C I A_{3}^{t}, \quad t \geq 1 \\
P T C_{3}^{t} & =p t c_{3}^{t-1} \text { or }\left[P T C_{3}^{t-1} \text { and not } H H_{2}^{t_{a}} \text { and not } h h_{2}^{t-1}, \quad t \geq 1,\right.
\end{aligned}
$$

where $\tau=2$ (respectively, $\tau=3$ ), if $c i_{3}^{0}=1$ (respectively, $c i_{3}^{0}=0$ ). Consider first the case $c i_{3}^{0}=1$. The activator protein will be turned on either at the first or second iterations: $C I A_{3}$ may become activated at $t=1$ because $P T C_{3}^{1}=0$, (if the permutation $\phi^{1}$ is such that $C I_{3}$ is updated before $C I A_{3}$ ). If $C I A_{3}$ is not actived at $t=1$, then it certainly is actived at $t=2$ (because $C I A_{3}^{1}=0$ implies $p t c_{3}^{1}=0$ and $P T C_{3}^{2}=0$ ). A similar argument shows that $C I R_{3}^{1,2}=0$.

Consider next the case $c i_{3}^{0}=0$ : we have $C I A_{3}^{1}=C I R_{3}^{1}=0$, and $C I A_{3}$ is turned on at the second or third iterations, by a similar argument as before. Therefore, the wingless gene is also expressed after $C I A_{3}$, at $T=1,2$ or 3 . 


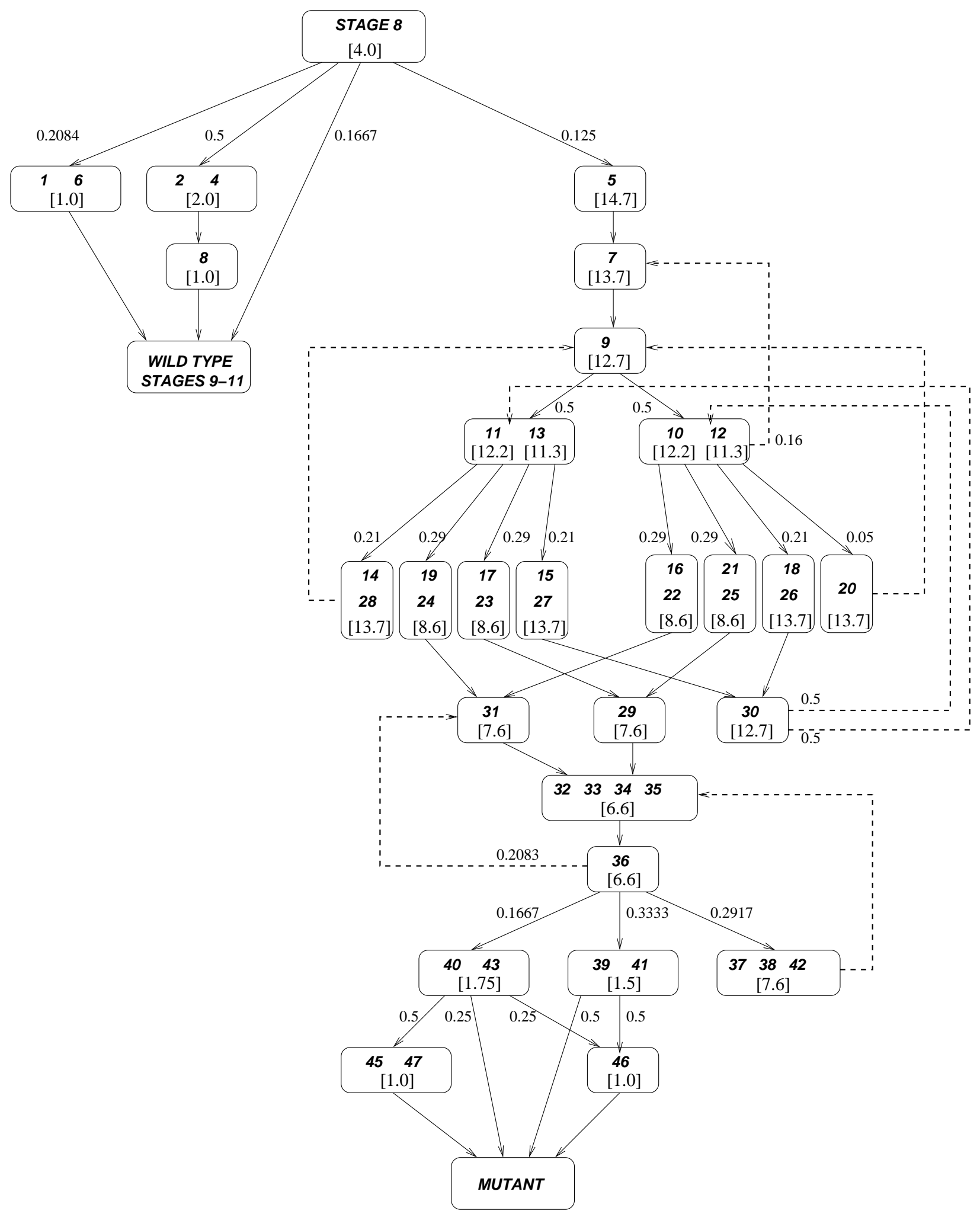

Figure 5: Robustness of the regulatory network modeled with the two-timescale algorithm. There are 48 states reachable from the wild type initial state. The arrows are labeled by the transition probabilities between states (if unlabeled, the probabilities are 1), and the expected times to absorption into the corresponding steady state are indicated between square brackets. 
Another necessary condition for convergence to wild type, is that

$$
w g_{4}^{0}=1 \text { or } e n_{1}^{0}=1 \text { or } c i_{4}^{0}=1
$$

Otherwise, the trajectories cannot converge to the wild type nor to the mutant steady states, In this case, the only possible steady state is a "lethal" state, where expression of PTC, ci, CIA and CIR is ubiqitous and all others are absent.

Proposition 6.1. Assume $w g_{3,4}^{0}=0, e n_{1}^{0}=0, p t c_{3}^{0}=1$ and $c i_{3,4}^{0}=0$. Then

(a) $w g_{3}=0$, for all $t \geq 0$.

(b) $P T C_{1}^{t}=1$ for all $t \geq 4$.

(c) $w g_{4}=0$ for infinitely many $t$.

Proof. Part (a) follows from Fact包), for any $T \geq 0$ :

$$
w g_{3}^{t}=0, \quad t \leq T \Rightarrow C I A_{3}=0,0<t \leq T+3 .
$$

Thus, by induction, $w g_{3}^{t}=0$ for $t \geq 0$.

Part (a) and Fact 4 imply $h h_{2}^{t}=0$ and $H H_{2}^{t}=0$ for all $t>0$, so that

$$
\begin{aligned}
p t c_{1}^{t} & =C I A_{1}^{t} \text { and not } C I R_{1}^{t} \text { and not } E N_{1}^{t} \\
P T C_{1}^{t} & =p t c_{1}^{t-1} \text { or } P T C_{1}^{t-1}, \quad t \geq 1 \\
C I A_{1}^{t} & =C I_{1}^{t_{a}} \text { and not } P T C_{1}^{t_{b}}, \quad t \geq 1 .
\end{aligned}
$$

If $p t c_{1}^{0}=1$, then it is clear that $P T C_{1}^{t}=1$ for all $t \geq 1$. Consider the case $p t c_{1}^{0}=0$. Then $P T C_{1}=0$ as long as $p t c_{1}=0$. Note that $c i_{4}^{0}=0$ implies $C I_{4}^{1}=0, C I A_{4}^{1}=0$ and also $w g_{4}^{1}=0$. Then $w g_{4}^{0,1}=0$ and $e n_{1}^{0,1}=0$ imply $E N_{1}^{1,2,3}=0$ and $C I_{1}^{2,3,4}=1$. So, either at $T=2$ or $T=3$, we will have $C I A_{1}^{T}=1$, and therefore also $p t c_{1}^{T}=1$ and $P T C_{1}^{T+1}=1$. Thus, $P T C_{4}^{t}=1$ for $t \geq 4$, proving part (b).

Finally, to argure by contradiction, suppose that $w g_{4}^{t}=1$ for $t \geq T_{a}$. Then by Corollary $4.3 P T C_{1}^{t}=1$ for $t \geq T_{b}>T_{a}$, which contradicts part (b). Hence, $w g_{4}$ cannot become permanently on.

By Proposition 4.1 together with Fact 2(b), a sufficient condition for convergence to wild type is

$$
w g_{4}^{0}=1, \quad p t c_{3}^{0}=1, \quad p t c_{1}^{0}=0, \quad c i_{1,3}^{0}=0 .
$$

Another sufficient condition (which allows the presence of cubitus in the third cell) is

$$
w g_{4}^{0}=1, \quad p t c_{3}^{0}=1, \quad P T C_{3}^{0}=1 .
$$

The argument in the proof of Proposition 4.5, shows that, if $P T C_{3}^{0}=1$ then $w g_{3}^{1}=0$. Then, by Proposition 4.4 it follows that $w g_{3}^{t}=0$ for all times.

In conclusion, while the wild type initial state allows for an ambiguity in the final states, we find that a remarkably minimal prepattern, consisting of $w g_{4}$ and $p t c_{3}$, is sufficient to guarantee the convergence to the wild type steady state. In other words, the initiation of two genes in two cells is enough to compensate for initiation delays in any and all other genes, irrespectively of the variations in individual synthesis and decay processes. This suggests a remarkable error correcting ability of the segment polarity gene control network. 


\section{Conclusions}

In summary, we proposed an intuitive and practical way of introducing stochasticity in qualitative models of gene regulation. We explored three possible ways of incorporating the variability of transcription, translation, post-translational modification and decay processes (see Table 5 for a comparison between the synchronous and three asynchronous algorithms). Applying our methods on a previously introduced model of the Drosophila segment polarity genes gave us new insights into the dynamics and function of the interactions among the segment polarity genes and, through it, into the robustness of the embryonic segmentation process. Our results suggest that unrestricted variability in synthesis/decay/transformation timescales can lead to a divergence from the wild type development process, with an expected divergence probability of $45 \%$. On the other hand, if the duration of post-translational transformations is consistently less than the duration of transcription, translation and mRNA/protein half-lives, the wild type steady state will be achieved with a high probability, despite significant variability in individual process durations. We find that a remarkably sparse prepattern is sufficient to ensure the convergence to the wild type steady state of these genes. This dual behavior, robustness to changes in the initial state but fragility with respect to temporal variability, is reminiscent of Highly Optimized Tolerance, a feature of highly structured, non-generic complex systems with robust, yet fragile external dynamics [Carlson and Doyle, 2002]. Similar robust-yet-fragile features have also been found in the of structure of diverse networks [Jeong et al., 2000 Jeong et al., 2001. Albert et al., 2000.

Table 5: Comparison of synchronous and asynchronous algorithms.

\begin{tabular}{lllll}
\hline & Synchronous & Totally Asynchronous & Random Order & Two-timescale \\
\hline \hline Assume & $\begin{array}{l}\text { Nodes are updated } \\
\text { at multiples } \\
\text { of the unit } \\
\text { time interval. }\end{array}$ & $\begin{array}{l}\text { The time between } \\
\text { updates is } \\
\text { perturbed in a } \\
\text { range } \pm \epsilon .\end{array}$ & $\begin{array}{l}\text { Each node is } \\
\text { updated at a randomly } \\
\text { selected point of the } \\
\text { unit time interval. }\end{array}$ & $\begin{array}{l}\text { In each time } \\
\text { interval proteins are } \\
\text { updated first, } \\
\text { then mRNAs. }\end{array}$ \\
\hline Update & $T^{k}=k$ & $T^{k}=T^{k-1}+1+\epsilon r^{k}$ & $T^{k}=k-1+\frac{1}{N} \phi^{k}$ & $T^{k}=k-1+\frac{1}{N} \phi_{t t}^{k}$ \\
& & & $\phi^{k}-$ node permutation & $\phi_{t t}^{k} \in\left(\phi_{\text {Prot }}^{k}, \phi_{m R N A}^{k}\right)$ \\
\hline Pros & Correctly identifies & Allows for unlimited & Does not depend & Allows separation \\
& all steady states. & variability in & on any perturbation & of post-translational \\
& Can be solved & process durations. & parameter $\epsilon$. & and pre-translational \\
& analytically. & & processes. \\
\hline Cons & $\begin{array}{l}\text { Dynamics is } \\
\text { unrealistic. }\end{array}$ & Can have unrealistically short transcription & Only useful when \\
& and translation times. & & process durations \\
& & & & can be separated. \\
\hline Results & $\begin{array}{l}\text { Prepattern errors } \\
\\
\text { can be corrected. }\end{array}$ & $\begin{array}{l}\text { Divergence from the wild type process is possible. } \\
\text { Cause: imbalance between two transcription factors. }\end{array}$ & $\begin{array}{l}\text { Development is stable if } \\
\text { PTC is prepatterned. }\end{array}$ \\
\hline
\end{tabular}

All our algorithms concur in suggesting that the divergence from wild type can be attributed to an imbalance between the two opposing Cubitus Interruptus transcription factors (CIA, CIR) in the posterior half of the parasegment. Thus the complementary regulation and pattern of these opposing transcription factors (Aza-Blanc and Kornberg 1999) is a vital requirement for the correct functioning of the segment polarity gene network. The totally asynchronous algorithm predicts that perturbations to the post-translational modification of Cubitus Interruptus can have effects as severe as mutations: a transient overexpression of CIR leads to the pattern with no segmentation, while transient expression of CIA and not CIR leads to the broad 
striped pattern. With the two timescale algorithm we find that the condition for the divergence from the wild type pattern is that, in the third cell of the parasegment, the post-translational modification of CI precedes the synthesis of the Patched protein. The biological realization of this condition appears unlikely, since PTC is documented as being ubiquitously expressed during cellularization (stage 5) [Taylor et al., 1993], while the post-translational modification of CI requires SMO that is only weakly expressed until stage 8 [Alcedo et al., 2000]. Our model predicts that if for any reason the PTC protein is absent in the period when the pair-rule proteins decay and the regulation between the segment polarity genes starts, the wild type expression pattern is unreachable."

Our methods combine the benefits of discrete-state models with a continuum in timescales. In the absence of quantitative information, we considered every possible timescale or update order, but as the twotimescale model demonstrates, existing information can be easily incorporated. We were able to describe the system in a rigorous mathematical way, to identify the relatively few types of behavior possible in the system (the attractors in state space) and to theoretically prove the convergence toward these states. Our results underscore that predictive mathematical modeling is possible despite the scarcity of quantitative information on gene regulatory processes.

\section{Acknowledgements}

The work of M.C. was supported in part by NIH Grants P20 GM64375 and Aventis. R.A. gratefully acknowledges an Alfred P. Sloan Research Fellowship. The work of E.D.S. was supported in part by NSF Grant CCR-0206789 and NIH Grants P20 GM64375 and R01 GM46383.

\section{A Additional Proofs}

Proof of Fact 마 For $T=0$, the statement follows directly from the model's equations and by using the assumptions A1-A2 repeatedly:

$$
W G_{4}^{1}=1, \quad e n_{1}^{1}=1, E N_{1}^{2}=1,
$$

as well as

$$
c i_{1}^{0,2}=0, \quad C I_{1}^{0,1,3}=0, \quad C I A_{1}^{0,1}=0, \quad C I R_{1}^{0,1}=0, \quad P T C_{1}^{0,1}=0 .
$$

We have (using (5))

$$
\begin{aligned}
p t c_{1}^{t} & =C I A_{1}^{t} \text { and not } C I R_{1}^{t} \text { and not } E N_{1}^{t} \\
P T C_{1}^{t} & =p t c_{1}^{t-1} \text { or }\left[P T C_{1}^{t_{b}} \text { and not } H H_{2}^{t_{c}}\right] \\
C I R_{1}^{t} & =C I_{1}^{t_{a}} \text { and } P T C_{1}^{t_{b}} \text { and not } H H_{2}^{t_{c}} \text { and not } h h_{2}^{t-1},
\end{aligned}
$$

so that we conclude

$$
p t c_{1}^{0,1,2}=0, \quad P T C_{1}^{2,3}=0, \quad C I R_{1}^{2,3}=0 .
$$

We next prove the Fact by induction. First note that, for any $t \geq 0$ :

$$
w g_{4}^{t}=1 \Rightarrow W G_{4}^{t+1}=1 \Rightarrow e n_{1}^{t+1}=1 \Rightarrow E N_{1}^{t+2}=1
$$


and this implies

$$
c i_{1}^{t+2}=0 \Rightarrow C I_{1}^{t+3}=0 .
$$

Now assume that the Fact holds for some $T \geq 1$ and that

$$
w g_{4}^{t}=1, \quad 0 \leq t \leq T .
$$

By the induction hypothesis, we know that

$$
C I_{1}^{t}=0,3 \leq t \leq T+2, \text { and } C I R_{1}^{t}=0,0 \leq t \leq T+2,
$$

By [18, $w g_{4}^{T}=1$ implies $C I_{1}^{T+3}=0$, and this together with $C I_{1}^{T+2}=0$ also guarantees that $C I R_{1}^{T+3}=$ 0 , as we wanted to show.

Proof of Fact 2. To prove part (a), assume that $w g_{3}^{t}=0$ for all $0 \leq t<T$, with $T \geq 1$. Since $e n_{2}^{0}=0$, then $E N_{2}^{0,1}=0$ and $h h_{2}^{1}=0, H H_{2}^{1,2}=0$. For $t \geq 3$ apply Fact 4 to obtain the desired value for $h h_{2}$ and $H H_{2}$. Note that $p t c_{3}^{0}=1$ implies $P T C_{3}^{1}=1$ and, together with $H H_{2}^{1,2}=0$, also $P T C_{3}^{2}=1$; then the value of $P T C_{3}$ follows from Fact 5 For $T \geq 2$, and using (5) and (8)), we have

$$
\begin{aligned}
& C I A_{3}^{t}=\operatorname{not} P T C_{3}^{t_{b}} \text { or } H H_{2}^{t_{c}} \text { or } h h_{2}^{t-1} \\
& C I R_{3}^{t}=\operatorname{PTC}_{3}^{s_{b}} \text { and not } H H_{2}^{s_{c}} \text { and not } h h_{2}^{t-1}
\end{aligned}
$$

so, the values for $h h_{2}, H H_{2}$ and $P T C_{3}$, indeed imply that $C I A_{3}^{t}=0$ and $C I R_{3}^{t}=1$, for $2 \leq t \leq T+3$.

To prove part (b), note that if $c i_{3}^{0}=0$, then also $c i_{3}^{0,1}=0$ and hence $C I A_{3}^{1}=0$. But now $C I A_{3}^{1}=0$ together with $w g_{3}^{0}=0$ immediately imply that $w g_{3}^{1}=0$, and therefore, the results in part (a) are valid for all $T \geq 0$.

\section{Fact 4.}

$$
h h_{2}^{T+2}=H H_{2}^{T+3}=w g_{3}^{T} \text { and } \operatorname{not} C I R_{2}^{T+2}, \text { for all } T \geq 0 .
$$

In particular, if $C I R_{2}^{t}=0$ for all $t \geq 3$, then $h h_{2}^{T+2}=H H_{2}^{T+3}=w g_{3}^{T}$, for all $T \geq 1$.

Proof. Given any $t \geq 0$ it is easy to see that

$$
\begin{aligned}
W G_{3}^{t+1} & =w g_{3}^{t} \\
e n_{2}^{t+1} & =W G_{1}^{t+1} \text { or } W G_{3}^{t+1} \equiv W G_{3}^{t+1}, \\
E N_{2}^{t+2} & =e n_{2}^{t+1}, \\
h h_{2}^{t+2} & =E N_{2}^{t+2} \text { and not } C I R_{2}^{t+2}, \\
H H_{2}^{t+3} & =h h_{2}^{t+2},
\end{aligned}
$$

where the equation for $e n_{2}^{t+1}$ follows from (4).

Fact 5. $P T C_{3}^{T}=1$ and $P T C_{3}^{T+1}=0$, for some $T>0$, only if $w g_{3}^{t}=1$ for some $t \in\{T-3, T-2\}$ (chosen according to the permutation $\phi^{T-2}$ ).

Proof. To see this, simply notice that

$$
\begin{aligned}
P T C_{3}^{T+1} & =p t c_{3}^{T} \text { or }\left[P T C_{3}^{T} \text { and not } H H_{2}^{t_{a}} \text { and not } H H_{4}^{t_{b}}\right] \\
& =p t c_{3}^{T} \text { or }\left[P T C_{3}^{T} \text { and not } H H_{2}^{t_{a}}\right] \\
& =p t c_{3}^{T} \text { or }\left[P T C_{3}^{T}\left(\text { and not } w g_{3}^{t_{a}-3} \text { or } C I R_{2}^{t_{a}-1}\right]\right.
\end{aligned}
$$

because from (5) $H H_{4}^{T}=0$, and by Fact 4 Note that $t_{a} \in\{T, T+1\}$, depending on the permutation $\phi^{T+1}$. So, for $P T C_{3}$ to vanish it is necessary that both $p t c_{3}^{T}=0$ and $w g_{3}^{t_{a}-3}=1$. 


\section{B Attractiveness of the wild type pattern}

Assuming that the trajectory is not of the form (17), the only accessible steady state is the wild type. In this case, to establish convergence of the trajectory, it is enough to show that each node attains a constant value after a finite number of iterates. And in fact, from Propositions 4.1, 4.4 and Corollaries 4.2, 4.3 all the nodes become fixed after at most $t$ iterates, as indicated:

$$
\begin{aligned}
& w g_{1,2}=0, W G_{1,2}=0, \quad t \geq 0, \quad \text { (4) } \\
& w g_{4}=1, W G_{4}=1, \quad t \geq 1, \quad \text { Proposition 4.1 } \\
& w g_{3}=0, W G_{3}=0, \quad t \geq 1, \quad \text { Proposition 4.4 } \\
& e n_{1}=1, E N_{1}=1, t \geq 2, \quad \text { Corollary 4.2 } \\
& e n_{2}=0, E N_{2}=0, t \geq 2, \quad \text { Corollary } 4.7 \\
& e n_{3,4}=0, E N_{3,4}=0, t \geq 0, \\
& h h_{1}=1, H H_{1}=1, \quad t \geq 3, \quad \text { Corollary } 4.2 \\
& h h_{2}=0, H H_{2}=0, t \geq 3, \quad \text { Corollary 4.7 } \\
& h h_{3,4}=0, H H_{3,4}=0, \quad t \geq 0, \\
& c i_{1}=0, C I_{1}=0, \quad t \geq 3, \quad \text { Corollary 4.2 } \\
& c i_{2}=1, C I_{2}=1, \quad t \geq 3, \quad \text { Corollary } 4.7 \\
& c i_{3,4}=1, C I_{3,4}=1, \quad t \geq 2, \quad \text { (7), (8) } \\
& \text { ptc } c_{1}=0, \quad P T C_{1}=0, \quad t \geq 0, \quad \text { Corollary 4.2 } \\
& C I A_{1}=0, C I R_{1}=0, \quad t \geq 4, \quad \text { Corollary 4.2 } \\
& C I A_{3}=0, C I R_{3}=1, \quad t \geq 2, \quad \text { Fact } 2 \\
& p t c_{3}=0, \quad P T C_{3}=1, t \geq 1, \quad \text { Fact } 2 \\
& C I A_{2}=1, C I R_{2}=0, t \geq 4, \quad C I_{2}=1 \\
& \text { ptc } c_{2}=1, \quad P T C_{2}=1, t \geq 5, \quad C I A_{2}=1, C I R_{2}=0, E N_{2}=0 \\
& C I A_{4}=1, C I R_{4}=0, t \geq 3, \quad w g_{4}=1 \\
& \text { ptc }_{4}=1, \quad P T C_{4}=1, \quad t \geq 5, \quad C I A_{4}=1, \quad C I R_{4}=0 .
\end{aligned}
$$

This is indeed a complete characterization of the wild type steady state.

Proof of Proposition 4.8. Let $P, A, C$ and $R(\leq L)$ denote the positions of $P T C_{3}, C I A_{3}, C I_{3}$ and $C I R_{3}$, respectively. Then, from (17) it is easy to see that

$P \in\{4,5,6, \ldots, L\}, A \in\{2,3,4, \ldots, P-1\}, C \in\{1,2,3, \ldots, A-1\}, R \in\{1, \ldots, P-1\} \backslash\{P, A, C\}$.

To derive a formula for $M$, we note that, for each pair of values $P, A$, the number of possible combinations of $C$ and $R$ is:

$$
\begin{array}{ll}
----\mathrm{A}-\mathrm{R}--\mathrm{P}: & (A-1)(P-1-A) \\
-\mathrm{R}--\mathrm{A}----\mathrm{P}: & (A-1)(A-2),
\end{array}
$$


respectively for sequences of the form $C A R P$ (top), or $C R A P$ and $R C A P$ (bottom). Therefore, summing over all posible $A$ and $P$ :

$$
\begin{aligned}
M_{L} & =\sum_{P=4}^{L} \sum_{A=2}^{P-1}[(A-1)(P-1-A)+(A-1)(A-2)] \\
& =\sum_{P=4}^{L} \sum_{A=2}^{P-1}(A-1)(P-3) \\
& =\frac{1}{2} \sum_{P=4}^{L}(P-3)(P-2)(P-1) \\
& =\frac{1}{2} \sum_{j=1}^{L-3} j(j+1)(j+2) .
\end{aligned}
$$

Now, for $L=4$,

$$
\frac{M_{L}(L-4) !}{L !}=\frac{1}{2} 3 ! \frac{0 !}{4 !}=\frac{1}{2} \frac{1}{4}=\frac{1}{8}
$$

Assume now that the equality is true for $L-1$ :

$$
\frac{M_{L-1}(L-5) !}{(L-1) !}=\frac{1}{8}
$$

Then

$$
\begin{aligned}
M_{L} & =M_{L-1}+\frac{1}{2}(L-3)(L-2)(L-1) \\
& =\frac{1}{8} \frac{(L-1) !}{(L-5) !}+\left(\frac{1}{2} L-3\right)(L-2)(L-1) \\
& =\frac{1}{8}(L-1)(L-2)(L-3)(L-4)+\left(\frac{1}{2} L-3\right)(L-2)(L-1) \\
& =(L-1)(L-2)(L-3)\left[\frac{1}{8}(L-4)+\frac{1}{2}\right] \\
& =(L-1)(L-2)(L-3)\left[\frac{1}{8} L\right]=\frac{1}{8} \frac{L !}{(L-4) !}
\end{aligned}
$$

just as we wanted to show.

\section{State aggregation in the Markov chain}

The tables below show the complete transition probabilities $p_{i j}$ (when not indicated, the transition probabilities are equal to 1). The states numbered 3 and 44 denote, respectively, the wild type and the mutant state. The initial condition was numbered 48 . The first table shows the complete transition probabilities at step 1 , from the wild type initial condition.

Thus the probability shown in the diagram for the transition from the initial state to the aggregated state 16 was obtained by adding $p_{48,1}+p_{48,6}=0.1667+0.0417=0.2084$. A more complex aggregation formula was used for the transition

$$
\begin{aligned}
1012 \rightarrow 1622:= & \frac{1}{2}\left(p_{10,16}+p_{10,22}\right)+\frac{1}{2}\left(p_{12,16}+p_{12,22}\right) \\
= & \frac{1}{2}(0.2083+0.0417+0.3+0.0333)=0.29165 \approx 0.29 .
\end{aligned}
$$


The normalization by $1 / 2$ is justified by the fact that the transition from 9 and 30 to either 10 or 12 is the same.

\begin{tabular}{|c|c|c|c|c|c|}
\hline \multicolumn{6}{|c|}{ From initial wild type state to: } \\
\hline 1 & 2 & 3 & 4 & 5 & 6 \\
\hline 0.1667 & 0.357 & 0.1667 & 0.125 & 0.125 & 0.0417 \\
\hline
\end{tabular}

\begin{tabular}{|c|c|c|c|}
\hline \multicolumn{4}{|c|}{ From 9 to: } \\
\hline 10 & 11 & 12 & 13 \\
\hline 0.25 & 0.25 & 0.25 & 0.25 \\
\hline
\end{tabular}

\begin{tabular}{|l|c|c|c|c|c|c|c|c|}
\hline & 7 & 16 & 18 & 20 & 21 & 22 & 25 & 26 \\
\hline From 10 to: & 0.2083 & 0.2083 & 0.125 & 0.0417 & 0.125 & 0.0417 & 0.125 & 0.125 \\
\hline From 12 to: & 0.1167 & 0.3 & 0.1167 & 0.05 & 0.1333 & 0.0333 & 0.2 & 0.05 \\
\hline
\end{tabular}

\begin{tabular}{|c|c|c|c|c|c|c|c|c|}
\hline & 14 & 15 & 17 & 19 & 23 & 24 & 27 & 28 \\
\hline From 11 to: & 0.2083 & 0.125 & 0.125 & 0.2083 & 0.125 & 0.0417 & 0.125 & 0.0417 \\
\hline From 13 to: & 0.1167 & 0.1167 & 0.1333 & 0.3 & 0.2 & 0.0333 & 0.05 & 0.05 \\
\hline
\end{tabular}

\begin{tabular}{|c|c|c|c|}
\hline \multicolumn{4}{|c|}{ From 29 or 31 to: } \\
\hline 32 & 33 & 34 & 35 \\
\hline 0.25 & 0.25 & 0.25 & 0.25 \\
\hline
\end{tabular}

\begin{tabular}{|c|c|c|c|}
\hline \multicolumn{4}{|c|}{ From 30 to: } \\
\hline 10 & 11 & 12 & 13 \\
\hline 0.25 & 0.25 & 0.25 & 0.25 \\
\hline
\end{tabular}

\begin{tabular}{|c|c|c|c|c|c|c|c|}
\hline \multicolumn{7}{|c|}{ From 36 to: } \\
\hline 31 & 37 & 38 & 39 & 40 & 41 & 42 & 43 \\
\hline 0.2083 & 0.125 & 0.125 & 0.2083 & 0.125 & 0.125 & 0.0417 & 0.0417 \\
\hline
\end{tabular}

\begin{tabular}{|c|c|c|c|}
\hline \multicolumn{4}{|c|}{ From 37 or 38 or 42 to: } \\
\hline 32 & 33 & 34 & 35 \\
\hline 0.25 & 0.25 & 0.25 & 0.25 \\
\hline
\end{tabular}

\begin{tabular}{|c|c|}
\hline \multicolumn{2}{|c|}{ From 39 or 41 to: } \\
\hline 44 & 46 \\
\hline 0.5 & 0.5 \\
\hline
\end{tabular}

\begin{tabular}{|c|c|c|c|}
\hline \multicolumn{4}{|c|}{ From 40 or 43 to: } \\
\hline 44 & 45 & 46 & 47 \\
\hline 0.25 & 0.25 & 0.25 & 0.25 \\
\hline
\end{tabular}

\section{References}

[Albert et al., 2000] Albert, R., Jeong, H. \& Barabási, A.-L., 2000. Error and attack tolerance in complex networks. Nature, 406, 378-382.

[Albert and Othmer, 2003] Albert, R. \& Othmer, H. G., 2003. The topology of the regulatory interactions predicts the expression pattern of the Drosophila segment polarity genes. J. Theor. Biol. 223, 1-18. 
[Alcedo et al., 2000] Alcedo, J., Zou, Y. \& Noll, M., 2000. Posttranscriptional regulation of smoothened is part of a self-correcting mechanism in the hedgehog signaling System. Molecular Cell 6, 457-465.

[Alon et al., 1999] Alon, U., Surette, M., Barkai, N. and Leibler, S., 1999. Robustness in Bacterial Chemotaxis. Nature 397, 168-171.

[Aza-Blanc \& Kornberg 1999] Aza-Blanc, P. \& Kornberg, T. B. (1999) Ci, a complex transducer of the Hedgehog signal, Trends in Genetics 15, 458-462.

[Bejsovec and Wieschaus, 1993] Bejsovec, A. \& Wieschaus, E., 1993. Segment polarity gene interactions modulate epidermal patterning in Drosophila embryos. Development 119, 501-517.

[Bernot et al., 2004] Bernot, G., Comet, J.-P., Richard, A. \& Guespin, J., 2004. Application of formal methods to biological regulatory networks: extending Thomas' asynchronous logical approach with temporal logic. J. Theor. Biol. 229, 339-347.

[Bertsekas and Tsitsiklis, 1989] Bertsekas, D.P. \& Tsitsiklis, J.N., 1989. Parallel and Distributed Computation, Numerical Methods. Prentice Hall, New Jersey.

[Bodnar, 1997] Bodnar, J. W., 1997. Programming the Drosophila Embryo. J. Theor. Biol. 188, 391-445.

[Cadigan et al., 1994] Cadigan, K. M., Grossniklaus, U. \& Gehring, W. J., 1994. Localized expression of sloppy paired protein maintains the polarity of Drosophila parasegments. Genes \& Dev. 8, 899-913.

[Cadigan \& Nusse 1997] Cadigan, K. M., \& Nusse, R. (1997) Wnt signaling: a common theme in animal development. Genes Dev. 11, 3286-3305.

[Carlson and Doyle, 2002] Carlson, J. M. \& Doyle, J., 2002. Complexity and robustness. Proc. Nat. Acad. Sci. 99, 2538-2545.

[Conant and Wagner, 2004] Conant, G. C.\& Wagner A., 2004. Duplicate genes and robustness to transient gene knock-downs in Caenorhabditis elegans. Proc. R. Soc. Lond. B Biol. Sci. 271, 89-96.

[Davidson et al., 2002] Davidson, E. H., et al., 2002. A genomic regulatory network for development. Science 295, 1669-1678.

[de Jong et al., 2004] de Jong, H. et al., 2004. Qualitative simulation of genetic regulatory networks using piecewise-linear models. Bull. Math. Bio. 66, 301-340.

[DiNardo et al., 1988] DiNardo, S., Sher, E., Heemskerk-Jongens, J., Kassis, J. A. \& O’Farrell, P. H., 1988. Two-tiered regulation of spatially patterned engrailed gene expression during Drosophila embryogenesis. Nature 332, 45-53.

[Eaton \& Kornberg 1990] Eaton, S. \& Kornberg, T. B. (1990) Repression of ci-D in posterior compartments of Drosophila by engrailed. Genes. Dev. 4, 1068-1077.

[Eldar et al., 2002] Eldar, A., Dorfman, R., Weiss, D., Ashe, H., Shilo, B.-Z. \& Barkai, N., 2002. Robustness of the BMP morphogen gradient in Drosophila embryonic patterning. Nature 419, 304-308.

[Feller, 1970] Feller, W., 1970. An Introduction to Probability Theory and its Applications (Third edition, revised). John Wiley \& Sons, New York, 1970. 
[Gallet et al., 2000] Gallet, A., Angelats, C., Kerridge, S. \& Thérond, P. P., 2000. Cubitus interruptusindependent transduction of the Hedgehog signal in Drosophila, Development 127, 5509-5522.

[Ghysen and Thomas, 2003] Ghysen, A. \& Thomas, R., 2003. The formation of sense organs in Drosophila: A logical approach. BioEssays 25, 802-807.

[Glass and Kauffman, 1973] Glass, L. \& Kauffman, S. A., 1973. The logical analysis of continuous, nonlinear biochemical control networks. J. Theor. Biol. 39, 103-129.

[Grossniklaus et al., 1992] Grossniklaus, U., Pearson, R. K. \& Gehring, W. J. (1992) The Drosophila sloppy paired locus encodes two proteins involved in segmentation that show homology with mammalian transcription factors.Genes Dev. 6, 1030-1051.

[Gursky et al., 2001] Gursky, V. V., Reinitz, J. \& Samsonov, A. M., 2001. How gap genes make their domains: An analytical study based on data driven approximations. Chaos 11, 132-141.

[Hidalgo and Ingham, 1990] Hidalgo, A. \& Ingham, P., 1990. Cell Patterning in the Drosophila segment: spatial regulation of the segment polarity gene patched. Development 110, 291-301.

[Hooper and Scott, 1992] Hooper, J. E. \& Scott, M. P., 1992. The Molecular Genetic Basis of Positional Information in Insect Segments. In: Early Embryonic Development of Animals (ed. Hennig, W.) $1-49$, Springer, Berlin.

[Ingham 1998] Ingham, P. W. (1998) Transducing hedgehog: the story so far, EMBO J. 17, 3505-3511.

[Ingham \& McMahon 2001] Ingham, P. W. \& McMahon, A. P. (2001) Hedgehog signaling in animal development: paradigms and principles, Genes Dev. 15, 3059-3087.

[Ingham et al., 1991] Ingham, P.W., Taylor, A. M. \& Nakano, Y., 1991. Role of the Drosophila patched gene in positional signaling. Nature 353, 184-187.

[Jeong et al., 2000] Jeong, H., Tombor, B., Albert, R., Oltvai, Z.N. \& Barabási, A.-L., 2000. The large-scale organization of metabolic networks. Nature 407, 651-654.

[Jeong et al., 2001] Jeong, H., Mason, S., Barabási, A.-L. \& Oltvai, Z. N., 2001. Lethality and centrality in protein networks. Nature 411, 41-42.

[Kauffman, 1993] Kauffman, S. A., 1993. The origins of Order. Oxford University Press, New York.

[Kauffman et al., 2003] Kauffman, S., Peterson, C., Samuelsson, B., Troein, C., 2003. Random Boolean network models and the yeast transcriptional network. Proc. Natl. Acad. Sci. USA. 100 14796-9.

[Martinez-Arias et al., 1988] Martinez-Arias, A., Baker, N. \& Ingham, P. W., 1988. Role of segment polarity genes in the definition and maintenance of cell states in the Drosophila embryo. Development $103,157-170$.

[Mendoza et al., 1999] Mendoza, L., Thieffry, D. \& Alvarez-Buylla, E. R., 1999. Genetic control of flower morphogenesis in Arabidopsis thaliana: a logical analysis. Bioinformatics 15, 593-606.

[Ohlmeyer \& Kalderon 1998] Ohlmeyer, J. T. \& Kalderon, D. (1998) hedgehog stimulates maturation of Cubitus interruptus into a labile transcriptional activator. Nature 396, 749-753. 
[Pfeiffer \& Vincent 1999] Pfeiffer, S. \& Vincent, J.-P. (1999) Signaling at a distance:Transport of Wingless in the embryonic epidermis of Drosophila. Cell \& Dev. Biol. 10, 303-309.

[Rao et al., 2002] Rao, C.V., Wolf, D.M. \& Arkin, A. P., 2002. Control, exploitation and tolerance of intracellular noise. Nature 420, 231-237.

[Reinitz and Sharp, 1995] Reinitz, J. \& Sharp, D. H., 1995. Mechanism of eve stripe formation. Mechanisms of Development 49, 133-158.

[Sánchez and Thieffry, 2001] Sánchez, L., \& Thieffry, D., 2001. A logical analysis of the Drosophila gapgene system. J. Theor. Biol. 211, 115-141.

[Schwartz et al., 1995] Schwartz, C., Locke, J., Nishida, C. \& Kornberg, T. B., 1995. Analysis of cubitus interruptus regulation in Drosophila embryos and imaginal disks. Development 121, 1625-1635.

[Tabata et al., 1992] Tabata, T., Eaton, S. \& Kornberg, T. B., 1992. The Drosophila hedgehog gene is expressed specifically in posterior compartment cells and is a target of engrailed regulation. Genes \& Dev. 6, 2635-2645.

[Taylor et al., 1993] Taylor, A. M., Nakano, Y., Mohler, J. \& Ingham, P. W., 1993. Contrasting distributions of patched and hedgehog proteins in the Drosophila embryo. Mechanisms of Development 42, 89-96.

[Thomas, 1973] Thomas, R., 1973. Boolean formalization of genetic control circuits. J. Theor. Biol. 42, 563-585.

[von Dassow et al., 2000] von Dassow, G., Meir., E., Munro, E. M., \& Odell, G. M., 2000. The segment polarity network is a robust developmental module. Nature 406, 188-192.

[Wolpert et al., 1998] Wolpert, L., Beddington, R., Brockes, J., Jessell, T., Lawrence, P., \& Meyerowitz, E., 1998. Principles of Development Current Biology Ltd., London.

[Yuh et al., 2001] Yuh, C. H., Bolouri, H., Bower, J. M. and Davidson, E. H., 2001. A logical model of cisregulatory control in a eukaryotic system. In: Computational Modeling of Genetic and Biochemical Networks (eds. Bower, J. M. and Bolouri, H.), 73-100, MIT Press, Cambridge, MA. 